TRANSACTIONS OF THE

AMERICAN MATHEMATICAL SOCIETY

Volume 275, Number 1, January 1983

\title{
ON STRONGLY INDEFINITE FUNCTIONALS WITH APPLICATIONS
}

BY

\author{
HELMUT HOFER
}

\begin{abstract}
Recently, in their remarkable paper Critical point theory for indefinite functionals, V. Benci and P. Rabinowitz gave a direct approach-avoiding finitedimensional approximations - to the existence theory for critical points of indefinite functionals. In this paper we develop under weaker hypotheses a simpler but more general theory for such problems. In the second part of the paper the abstract results are applied to a class of resonance problems of the Landesman and Lazer type, and moreover they are illustrated by an application to a wave equation problem.
\end{abstract}

0. Introduction. This paper is devoted to the critical point theory of strongly indefinite functionals in a real Hilbert space $H$. Here a strongly indefinite functional is a $C^{1}$-map $H \rightarrow \mathbf{R}$ which is neither bounded from above or below not even modulo a finite-dimensional subspace. Such functionals arise for example in the study of periodic solutions of the one-dimensional wave equation, in the study of periodic solutions of Hamiltonian systems of ordinary differential equations, in the existence theory for systems of elliptic equations, etc.

To find the critical points of strongly indefinite functionals different methods were used. For example, Rabinowitz studied the restrictions of the functional to finite-dimensional subspaces, [1,2], and then he tried to find the critical points of the original problem by passing to the limit for a suitable subsequence of the critical points of the restricted problems. Amann [3] reduced the indefinite functionals by means of a saddlepoint-reduction to a finite-dimensional problem. Of course, this is only possible under some strong hypotheses. Under certain assumptions the functional can be replaced by a dual variational problem, having some better properties. (See for example [4 or 5].) The first direct approach was given by Benci and Rabinowitz [6]. The essential in their paper is the observation that more detailed information about the special form of the deformations (a substitute for the gradient flow) associated to the functional is needed. They constructed deformations having special representations by solving appropriate differential equations approximately by time discretisation. This method has some disadvantages because one needs strong hypotheses in order to get the approximations uniformly on bounded sets.

A main part of our paper will be the construction of flows and deformations having special representations by solving appropriate differential equations exactly

Received by the editors March 30, 1981 and, in revised form, November 30, 1981.

1980 Mathematics Subject Classification. Primary 58E05; Secondary 47H25, 35L05.

Key words and phrases. Critical point theory, resonance problem, wave equation.

(C) 1983 American Mathematical Society $0002-9947 / 82 / 0000-0824 / \$ 08.50$ 
and the study of their topological properties. Moreover, we shall develop a variant of an "intersection theory" for infinte-dimensional sets in Hilbert space.

Some of the results contained in this paper can be generalized. A few generalizations are contained in a preliminary version of this paper having a different title. For the sake of simplicity and readability we will not carry this out. I am indebted to the referee and P. Rabinowitz for some suggestions in this direction. Moreover I want to thank H. Amann and P. Hess for stimulating discussions.

In order to make the difficulties which arise clear to nonspecialists, let us recall the min-max- or max-min-principle (MMP). Here the interested reader should also consult the paper of R. Palais [7].

The MM-principle. Let $\Phi \in C^{1}(H, \mathbf{R})$ and define for real numbers $d$ and $c$ the sets $\Phi_{d}=\Phi^{-1}([d,+\infty)), \Phi^{c}=\Phi^{-1}((-\infty, c])$, and $\Phi_{d}^{c}=\Phi^{c} \cap \Phi_{d}$.

Definition 1. A MM-theory for $\Phi$ is a triple $t(\Phi)=(D, S, c)$, where

(D) $D$ is a family of homeomorphisms $\sigma: H \rightarrow H$, such that for all $d \in \mathbf{R}$ being not a critical value of $\Phi$ there exist $\varepsilon>0$ and $\sigma \in D$ with $\sigma\left(\Phi_{d-\varepsilon}\right) \subset \Phi_{d+\varepsilon}$.

(S) $S$ is a family of subsets of $H$ with $D(S) \subset S$, i.e. $\sigma(\xi) \in S$ for all $\sigma \in D$ and all $\xi \in S$.

(c) $c$ is a real number verifying $c=\sup _{\xi \in S} \inf \Phi(\xi)$.

Of course, it is not clear in general if such a theory exists for a critical value of $\Phi$. More precisely we have the following important result (see also [7]) which is an easy consequence of the definition of $t(\Phi)$.

TheOREM (MMP). Let $\Phi \in C^{1}(H, \mathbf{R})$ and assume $t(\Phi)=(D, S, c)$ is a MM-theory for $\Phi$. Then $c$ is a critical value.

Proof. Arguing indirectly, we assume that $c$ is not a critical value. By (c) $c=\sup _{\xi \in S} \inf \Phi(\xi)$ is a real number. Using (D) we find $\varepsilon>0$ and $\sigma \in D$ with $\sigma\left(\Phi_{c-\varepsilon}\right) \subset \Phi_{c+\varepsilon}$. By the definition of $c \in \mathbf{R}$ there exists $\xi \in S$ with $\xi \subset \Phi_{c-\varepsilon}$. Then $\xi^{*}=\sigma(\xi) \in S$ by (S) and $\xi^{*} \subset \Phi_{c+\varepsilon}$ by (D). This implies the contradiction $c \geqslant$ inf $\Phi\left(\xi^{*}\right) \geqslant c+\varepsilon$.

Using this principle the program is clear. In order to find critical points of a given functional $\Phi$ we have to construct such triples $t(\Phi)$, which describe a critical value of $\Phi$.

First of all we will speak about the existence of a family $D$ verifying (D) and give a condition which guarantees its existence.

Definition 2. A functional $\Phi \in C^{1}(H, \mathbf{R})$ verifies the weak Palais-Smale condition if the following holds:

(WPS) If for some sequence $\left(u_{n}\right) \subset H$ we have $\Phi^{\prime}\left(u_{n}\right) \rightarrow 0$ and $\Phi\left(u_{n}\right) \rightarrow d \in \mathbf{R}$, then $d$ is a critical value.

Observe that (WPS) is a variant of the condition (C) introduced by R. Palais and S. Smale. It is well known that (WPS) is sufficient for the existence of such a family $D$, and that the maps $\sigma$ can be chosen in such a way that they are homotopic to Id by a homotopy $\eta:[0,1] \times H \rightarrow H$ having the property that for all $t \in[0,1]$ the maps $\eta^{t}=\eta(t, \cdot): H \rightarrow H$ are homeomorphisms and, in addition, the maps $t \rightarrow \Phi\left(\eta^{t}(u)\right)$ are nondecreasing for all $u \in H$. These two facts are of fundamental importance for practical purposes. 
Next, some remarks about the construction of a family $S$. Suppose a family $D$ of homeomorphisms verifying (D) and the additional hypotheses from above is given. Further assume that $D \circ D \subset D$, i.e. $\sigma \circ \tilde{\sigma} \in D$ for all $\sigma, \tilde{\sigma} \in D$. Suppose there exist subsets $\xi, \xi^{*}$ of $H$ with

$$
\begin{gathered}
\inf \Phi(\xi)>-\infty, \quad \sup \Phi\left(\xi^{*}\right)<+\infty \text { and } \\
\sigma(\xi) \cap \xi^{*} \neq \varnothing \quad \text { for all } \sigma \in D .
\end{gathered}
$$

In that case we define

$$
S=\{\sigma(\xi) \mid \sigma \in D\} \quad \text { and } \quad c=\sup _{\xi \in S} \inf \Phi(\xi) .
$$

Observe that $D(S) \subset S$ since $D \circ D \subset D$. The important thing here is the intersection property of $\xi$ and $\xi^{*}$, i.e. the fact that $\sigma(\xi) \cap \xi^{*} \neq \varnothing$ for all $\sigma$. In practice, in order to show such an intersection property, it is important that $D$ verifies the additional hypotheses from above. Then making use of tools of algebraic topology, for example using topological intersection theory or degree theory, one tries to prove this property.

At this point the difficulty in the study of strongly indefinite functionals comes in. In general, the sets $\xi$ and $\xi^{*}$ are infinite-dimensional and the invariants of algebraic topology give no tool to prove this intersection property directly. This is due to the fact that for infinite-dimensional sets the (co-)homology etc., is of ten trivial since it is a homotopy invariant with respect to all continuous maps. (For example, the unit sphere of an infinite-dimensional Hilbert space is contractible to a point, which is of course wrong in the finite-dimensional case.)

Here of course it is useful to allow only special continuous maps having representations depending on the functional $\Phi$ and to show that topological invariants exist to build up something like an intersection theory for the restricted family of admissible maps.

In the second part of this paper we use the theory developed in the first part to study the ranges of a class of potential operators $\Phi^{\prime}: H \rightarrow H$. This class arises in problems of the Landesman and Lazer type (see [8 and 9]). $\Phi^{\prime}$ will have the decomposition $\Phi^{\prime}=A+B$, where $A$ is a bounded linear selfadjoint operator with a nontrivial kernel $N(A)$ and $B$ is a continuous potential operator verifying some compactness assumptions. The abstract results for the Landesman and Lazer type problems will then be illustrated by an application to a wave equation problem. However, the theory also applies to the problem of finding periodic solutions of Hamiltonian systems or to the existence theory for elliptic systems.

\section{THE THEORY FOR STRONGLY INDEFINITE FUNCTIONALS}

1. Flows associated to a class of strongly indefinite functionals and a related group $(M, \circ)$ of deformations. Denote by $H=(H,(\cdot, \cdot))$ a real Hilbert space. To motivate the following definitions assume that the gradient $\Phi^{\prime}$ of $\Phi \in C^{1}(H, \mathbf{R})$ admits the decomposition $\Phi^{\prime}=A+B$, where $A: H \rightarrow H$ is a bounded linear selfadjoint operator and $B: H \rightarrow H$ is continuous. Suppose that $B$ is locally Lipschitz continuous and eventually verifying some growth conditions such that the flow $\eta$ associated to

$$
\dot{\eta}_{u}=\Phi^{\prime}\left(\eta_{u}\right), \quad \eta_{u}(0)=u
$$


exists globally. In that case we find that $\eta$ admits the representation

$$
\eta(t, u)=\exp (t A) u+\int_{0}^{t}(\exp ((t-s) A) B(\eta(s, u))) d s .
$$

If for example $Q: H \rightarrow H$ is an orthogonal projection commuting with $A$ such that $Q B$ is compact and $\eta$ maps bounded sets into bounded sets we infer that the map $(t, u) \rightarrow Q \int_{0}^{t} \exp ((t-s) A) B(\eta(s, u)) d s$ is compact, since $\exp ((t-s) A)$ and $Q$ commute for all $t, s$. Moreover $\exp (t A)$ is an element in the smallest closed subalgebra containing $A$ and Id in the algebra of all bounded linear operators $H \rightarrow H$.

Let us introduce the following notions. For $X, Y$ metric spaces define

$$
\begin{gathered}
C(X, Y)=\{f: X \rightarrow Y \mid f \text { is continuous }\}, \\
C_{b}(X, Y)=\{f \in C(X, Y) \mid f \text { maps bounded sets into bounded sets }\}, \\
K(X, Y)=\left\{f \in C_{b}(X, Y) \mid f \text { maps bounded sets into relatively compact sets }\right\} .
\end{gathered}
$$

Let

$$
\begin{gathered}
L(H)=\{T: H \rightarrow H \mid T \text { is a bounded linear operator }\}, \\
G(H)=\{T \in L(H) \mid T \text { is bijective }\} .
\end{gathered}
$$

For a given selfadjoint operator $A \in L(H)$ introduce

$$
\begin{gathered}
{[A]=\text { smallest closed subalgebra of } L(H) \text { containing } A \text { and Id, }} \\
\qquad G_{A}(H)=[A] \cap G(H),
\end{gathered}
$$

both equipped with the induced metric from $L(H)$. Note that $[A]$ and $G_{A}(H)$ are commutative. By $B_{r}$ we denote the open ball in $H$ with centre 0 and radius $r$.

Assume $A \in L(H)$ is selfadjoint and $Q \in L(H)$ an orthogonal projection commuting with $A$. Now we introduce a linear subspace $\Sigma=\Sigma(H, A, Q)$ of $C^{1}(H, \mathbf{R})$.

Definition 3. Let $A$ and $Q$ be as above. The linear subspace $\Sigma$ consists of all $\Phi \in C^{\prime}(H, \mathbf{R})$ with the composition $\Phi^{\prime}=\bar{A}+\bar{B}$, where $\bar{A} \in[A], \bar{B} \in C(H, H)$, and $Q \bar{B} \in K(H, H)$.

For given $A$ and $Q$, as above, we will define a group $(M, \circ)$ of parameter-depending homeomorphisms which will be of fundamental importance for the study of functionals $\Phi \in \Sigma$.

Definition 4. Let $A$ and $Q$ be as above. Denote by $M=M(H, A, Q)$ the set consisting of all maps $\eta: \mathbf{R} \times H \rightarrow H$ verifying

$$
\eta(t, u)=\gamma(t, u) u+C(t, u)
$$

for all $(t, u) \in \mathbf{R} \times H$, with $\gamma \in K\left(\mathbf{R} \times H, G_{A}(H)\right), \gamma(0, u)=$ Id for all $u \in H$, $C \in C_{b}(\mathbf{R} \times H, H), Q C \in K(\mathbf{R} \times H, H), C(0, u)=0$ for all $u \in H$. For fixed $t \in \mathbf{R}$ the map $\eta^{t}: H \rightarrow H: u \rightarrow \eta(t, u)$ is a homeomorphism such that the map $(t, u) \rightarrow\left(\eta^{t}\right)^{-1}(u)$ is in $C_{b}(\mathbf{R} \times H, H)$.

Observe that $\eta^{0}=\mathrm{Id}$ and that $\eta \in C_{b}(\mathbf{R} \times H, H)$.

For $\eta, \chi \in M$ we define maps $\eta \circ \chi$ and $\eta^{*}$ by

$$
\begin{aligned}
& (\eta \circ \chi)(t, u)=\eta^{t}\left(\chi^{t}(u)\right) \quad \text { for all }(t, u) \in \mathbf{R} \times H, \\
& \eta^{*}(t, u)=\left(\eta^{t}\right)^{-1}(u) \quad \text { for all }(t, u) \in \mathbf{R} \times H .
\end{aligned}
$$


First of all we shall show that $\eta^{*}, \eta \circ \chi \in M$. Assume $\eta$ and $\chi$ have the following representations:

$$
\begin{aligned}
\eta(t, u) & =\gamma(t, u) u+C(t, u), \\
\chi(t, u) & =\delta(t, u) u+D(t, u)
\end{aligned}
$$

Then

$$
\begin{aligned}
(\eta \circ \chi)(t, u) & =\gamma(t, \chi(t, u)) \delta(t, u) u+(C(t, \chi(t, u))+\gamma(t, \chi(t, u)) D(t, u)) \\
& =: \tau(t, u) u+E(t, u) \quad \text { for all }(t, u) \in \mathbf{R} \times H .
\end{aligned}
$$

Since $G_{A}(H)$ is a group $\tau \in K\left(\mathbf{R} \times H, G_{A}(H)\right)$ and clearly $E \in C_{b}(\mathbf{R} \times H, H)$ by the boundedness of $\chi, C, D$. Observing that $Q$ commutes with the elements in $[A]$ we find that $Q E$ is compact. Of course $(\eta \circ \chi)^{\mathrm{t}}$ is a homeomorphism and the map $(t, u) \rightarrow\left((\eta \circ \chi)^{t}\right)^{-1}(u)$ is in $C_{b}(\mathbf{R} \times H, H)$. Hence “。" defines a map $M \times M \rightarrow M$. Next we will show that $\eta^{*} \in M$. Let $u=\eta(t, v)$. We have

$$
\begin{aligned}
\eta^{*}(t, u) & =v=(\gamma(t, v))^{-1}(u)-(\gamma(t, v))^{-1} C(t, v) \\
& =\gamma\left(t, \eta^{*}(t, u)\right)^{-1}(u)-\gamma\left(t, \eta^{*}(t, u)\right)^{-1} C\left(t, \eta^{*}(t, u)\right) \\
& =: \gamma^{*}(t, u) u+C^{*}(t, u) .
\end{aligned}
$$

Using that $\eta^{*} \in C_{b}(\mathbf{R} \times H, H)$ one easily sees that $\eta^{*} \in M$. Obviously, $\eta \circ \eta^{*}=e$ $=\eta^{*} \circ \eta$, where $e(t, u)=u$ for all $(t, u)$. Observe that $e \in M$. Thus we have proved the following lemma

Lemma 1. $(M, \circ)$ is a group. $e$ is the neutral element and the inverse of a given $\eta \in M$ is $\eta^{*} \in M$.

In the following we will show that $M$ contains maps having special properties. Fix a selfadjoint $A \in L(H)$ and an orthogonal projection $Q \in L(H)$ commuting with $A$. We will use the abbreviations $\Sigma=\Sigma(H, A, Q)$ for $M=M(H, A, Q)$.

TheOrem 1. Assume $\Phi \in \Sigma$. Then for arbitrarily given open sets $V$ and $W$ in $H$ with $\operatorname{cl}(V) \cap \operatorname{cl}(W)=\varnothing$ and $\left\|\Phi^{\prime}(u)\right\| \geqslant \varepsilon$ for all $u \in V$ there exists a flow $\eta \in M$, continuously differentiable with respect to $t \in \mathbf{R}$ such that:

(i) $d \Phi\left(\eta^{t}(u)\right) / d t \geqslant \frac{1}{2} \beta\left(\eta^{t}(u)\right)\left\|\Phi^{\prime}\left(\eta^{t}(u)\right)\right\|$ for all $(t, u) \in \mathbf{R} \times H$, where $\beta: H \rightarrow$ $[0,1]$ is a continuous map with $\beta \mid W \equiv 0$ and $\beta \mid V \equiv 1$.

(ii) $\eta^{t} \mid \operatorname{cl}(W)=$ Id for all $t \in \mathbf{R}$.

(iii) $\|u\| \exp (-|t|) \leqslant\left\|\eta^{t}(u)\right\| \leqslant(\|u\|+1) \exp (|t|)$ for all $(t, u) \in \mathbf{R} \times H$.

Proof. Let $\Phi \in \Sigma$ such that $\Phi^{\prime}=L+B$, where $L \in[A], B \in C(H, H)$, and moreover $Q B \in K(H, H)$. By a standard partition of unity argument we find a locally Lipschitz continuous map $B^{*}: H \rightarrow H$ such that $Q B^{*}$ is compact and

$$
\left\|B u-B^{*} u\right\| \leqslant \varepsilon / 8 \text { for all } u \in H .
$$

Define $C: H \rightarrow H$ by $C u=L u+B^{*} u$. For $u \in \operatorname{cl}(V)$ we have $\|C u\| \geqslant \frac{7}{8} \varepsilon$. We find an open neighbourhood $U$ of $\operatorname{cl}(V)$ such that $\operatorname{cl}(U) \cap \operatorname{cl}(W)=\varnothing$ and $\|C u\| \geqslant \frac{3}{4} \varepsilon$ for all $u \in U$. There exists a locally Lipschitz continuous function $\beta: H \rightarrow[0,1]$ such 
that $\beta \mid \operatorname{cl}(U) \equiv 1$ and $\beta \mid \operatorname{cl}(W) \equiv 0$. Define a locally Lipschitz continuous operator $G: H \rightarrow H$ by

$$
G u= \begin{cases}\beta(u)\|C u\|^{-1} C u & \text { if } u \in U, \\ 0 & \text { if } u \in H \backslash U .\end{cases}
$$

Clearly this is well defined since $\|C u\| \geqslant \frac{3}{4} \varepsilon$ for all $u \in U$. One easily obtains the following estimate:

$$
\left(\Phi^{\prime}(u), G u\right) \geqslant \frac{1}{2} \beta(u)\left\|\Phi^{\prime}(u)\right\| \quad \text { for all } u \in H .
$$

We solve the ordinary differential equation

$$
\dot{\eta}_{u}=G \eta_{u}, \quad \eta_{u}(0)=u \quad \text { for } u \in H .
$$

Since $G$ is locally Lipschitz continuous and $\|G u\| \leqslant 1$ for all $u \in H$ we have by the basic existence theorem global existence on $\mathbf{R}$. We obtain a flow $\eta: \mathbf{R} \times \boldsymbol{H} \rightarrow \boldsymbol{H}$ by $\eta(t, u):=\eta_{u}(t)$. Moreover we infer from (4) the estimate

(5) $\|u\| \exp (-|t|) \leqslant\|\eta(t, u)\| \leqslant(\|u\|+1) \exp (|t|) \quad$ for all $(t, u) \in \mathbf{R} \times H$.

Hence we have established (iii). (i) follows from

$$
\frac{d}{d t} \Phi\left(\eta^{t}(u)\right) \geqslant \frac{1}{2} \beta\left(\eta^{t}(u)\right)\left\|\Phi^{\prime}\left(\eta^{t}(u)\right)\right\|
$$

and (ii) since $\dot{\eta}^{t}(u)=0, \eta(0, u)=u$ for $u \in \operatorname{cl}(W)$. It remains to show that $\eta \in M$. Define $\alpha \in C_{b}\left(\mathbf{R} \times H, \mathbf{R}^{+}\right)$and $\alpha_{L} \in K\left(\mathbf{R}^{2} \times H, G_{A}(H)\right)$ by

$$
\begin{gathered}
\alpha(t, u)= \begin{cases}\beta\left(\eta^{t}(u)\right)\left\|C \eta^{t}(u)\right\|^{-1} & \text { if } \eta^{t}(u) \in U, \\
0 & \text { if } \eta^{t}(u) \in H \backslash U,\end{cases} \\
\alpha_{L}(t, s, u)=\exp \left(\left(\int_{0}^{t-s} \alpha(a+s, u) d a\right) L\right) .
\end{gathered}
$$

The compactness of $\alpha_{L}$ follows immediately from the continuity of exp: $[A] \rightarrow G_{A}(H)$ and the compactness of the map

$$
\mathbf{R}^{2} \times H \rightarrow \mathbf{R} L \subset[A]:(t, s, u) \rightarrow\left(\int_{0}^{t-s} \alpha(a+s, u) d a\right) L,
$$

since the map is of class $C_{b}$ and the range is a subset of a one-dimensional Banach space. Let $\hat{B}: \mathbf{R} \times H \rightarrow H$ be defined by

$$
\hat{B}(s, u)=B^{*}(\eta(s, u)) .
$$

Clearly $\hat{B}$ is continuous and $Q \hat{B}$ is compact. Define $\chi: \mathbf{R} \times H \rightarrow H$ by

$$
\begin{aligned}
\chi(t, u) & =\alpha_{L}(t, 0, u) u+\int_{0}^{t}\left(\alpha_{L}(t, s, u)(\alpha(s, u) \hat{B}(s, u))\right) d s \\
& =: \gamma(t, u) u+E(t, u) .
\end{aligned}
$$

Obviously $\gamma \in K\left(\mathbf{R} \times H, G_{A}(H)\right)$ and $Q E \in K(\mathbf{R} \times H, H)$, since $Q$ and $\alpha_{L}(t, s, u)$ commute for all $(t, s, u)$ and the map $\mathbf{R}^{2} \times H \rightarrow H:(t, s, u) \rightarrow \alpha_{L}(t, s, u)$. $(\alpha(s, u) Q \hat{B}(s, u))$ is compact. Moreover $\chi(\cdot, u)$ is continuously differentiable for fixed $u \in H$ and solves (4). By uniqueness $\chi=\eta$. By the preceding discussion $\eta \in M$. 
As a corollary we obtain a deformation lemma which is a refinement of the usual deformation lemmas and which states, in addition, that the deformation can be chosen in $M$.

Corollary 1. Let $\Phi \in \Sigma$ and suppose there exist numbers $d_{1}, d_{2} \in \mathbf{R}, d_{1} \leqslant d_{2}$, $r \geqslant s>0$, and $\alpha>0$ such that

$$
\begin{gathered}
r \geqslant(s+1) \exp \left(\frac{2}{\alpha}\left(d_{2}-d_{1}\right)\right) \quad \text { and } \\
\left\|\Phi^{\prime}(u)\right\| \geqslant \alpha \quad \text { for all } u \in \Phi_{d_{1}}^{d_{2}} \cap\left(B_{r} \backslash B_{s}\right) .
\end{gathered}
$$

Then the following alternative holds: Either there exists a sequence $\left(u_{n}\right) \subset \Phi_{d_{1}}^{d_{2}} \cap B_{s}$ with $\Phi^{\prime}\left(u_{n}\right) \rightarrow 0$ or there exists a flow $\eta \in M$, continuously differentiable with respect to $t \in \mathbf{R}$, such that

(i) $d \Phi\left(\eta^{t}(u)\right) / d t \geqslant 0$ for all $(t, u) \in \mathbf{R} \times H$.

(ii) $\eta\left(t_{\infty}, B_{s} \cap \Phi_{d_{1}}\right) \subset \Phi_{d_{2}}$ where $t_{\infty}$ is large enough.

REMARK. Corollary 1 will be very important for the proof of a perturbation result in $\$ 3$.

Proof. Let $V=B_{r} \cap \operatorname{int}\left(\Phi_{d_{1}}^{d_{2}}\right)$ and $W=H \backslash \operatorname{cl}\left(B_{r+1}\right)$. Assume there exists no sequence $\left(u_{n}\right) \subset \Phi_{d_{1}}^{d_{2}} \cap B_{s}$ with $\Phi^{\prime}\left(u_{n}\right) \rightarrow 0$. Then we find $\varepsilon \in(0, \alpha)$ with $\left\|\Phi^{\prime}(u)\right\| \geqslant \varepsilon$ for all $u \in \Phi_{d_{1}}^{d_{2}} \cap B_{r}$. Now applying Theorem 1 we find $\eta \in M$ with

$$
\begin{aligned}
& \frac{d}{d t} \Phi\left(\eta^{t}(u)\right) \geqslant \frac{1}{2} \beta\left(\eta^{t}(u)\right)\left\|\Phi^{\prime}\left(\eta^{t}(u)\right)\right\| \quad \text { for all }(t, u) \in \mathbf{R} \times H, \\
& \left\|\eta^{t_{2}}(u)\right\| \leqslant\left(\left\|\eta^{t_{1}}(u)\right\|+1\right) \exp \left(t_{2}-t_{1}\right) \text { for all } u \in H, t_{1} \leqslant t_{2} .
\end{aligned}
$$

Now let $u \in B_{s} \cap \Phi_{d_{1}}^{d_{2}}$. Assume first that $\eta^{t}(u) \in B_{r} \cap \Phi_{d_{1}}^{d_{2}}$ for all $t \in\left[0, t_{\infty}\right]$ where $t_{\infty}=\frac{2}{\varepsilon}\left(d_{2}-d_{1}\right)$. Then

$$
\Phi\left(\eta^{t_{\infty}}(u)\right) \geqslant d_{1}+\int_{0}^{t} \frac{\varepsilon}{2} d t=d_{1}+d_{2}-d_{1}=d_{2} .
$$

If this is not the case we have, for some $t^{\prime} \in\left[0, t_{\infty}\right], \eta^{t^{\prime}}(u) \notin B_{r}$ or $\eta^{t^{\prime}}(u) \notin \Phi_{d_{1}}^{d_{2}}$. In the second case we must have $\Phi\left(\eta^{t^{\prime}}(u)\right)>d_{2}$ and conclude that

$$
\Phi\left(\eta^{t_{\infty}}(u)\right) \geqslant d_{2} \quad \text { if } \eta^{t^{\prime}}(u) \notin \Phi_{d_{1}}^{d_{2}} \text { for some } t^{\prime} \in\left[0, t_{\infty}\right] .
$$

Hence we may assume that $\eta^{t^{\prime}}(u) \notin B_{r}$ for some $t^{\prime} \in\left[0, t_{\infty}\right]$ but $\eta^{t}(u) \in \Phi_{d_{1}}^{d_{2}}$ for all $t \in\left[0, t_{\infty}\right]$. Define real numbers $t_{1}$ and $t_{2}$ by

$$
\begin{aligned}
& t_{2}=\inf \left\{t \in\left[0, t_{\infty}\right] \mid \eta^{t}(u) \notin B_{r}\right\}, \\
& t_{1}=\sup \left\{t \in\left[0, t_{2}\right] \mid \eta^{t}(u) \in B_{s}\right\} .
\end{aligned}
$$

We find

$$
\Phi\left(\eta^{t_{\infty}}(u)\right) \geqslant d_{1}+\int_{t_{1}}^{t_{2}} \frac{d}{d t} \Phi\left(\eta^{t}(u)\right) d t \geqslant d_{1}+\frac{\alpha}{2}\left(t_{2}-t_{1}\right) .
$$

Let us estimate $t_{2}-t_{1}$. By the definition of $t_{1}$ and $t_{2}$ we must have

$$
\left\|\eta^{t_{2}}(u)\right\|=r \text { and }\left\|\eta^{t_{1}}(u)\right\|=s
$$


By the properties of $\eta$

$$
r \leqslant(s+1) \exp \left(t_{2}-t_{1}\right)
$$

or equivalently

$$
t_{2}-t_{1} \geqslant \ln (r /(s+1)) \text {. }
$$

By our hypothesis

$$
t_{2}-t_{1} \geqslant \frac{2}{\alpha}\left(d_{2}-d_{1}\right)
$$

which implies

$$
\Phi\left(\eta^{t_{\infty}}(u)\right) \geqslant d_{1}+\frac{\alpha}{2} \frac{2}{\alpha}\left(d_{2}-d_{1}\right)=d_{2} .
$$

Since $u$ was arbitrarily chosen in $\Phi_{d_{1}}^{d_{2}} \cap B_{s}$ we conclude that

$$
\eta\left(t_{\infty}, \Phi_{d_{1}}^{d_{2}} \cap B_{s}\right) \subset \Phi_{d_{2}} \text {. }
$$

Combining this with $\eta\left(t_{\infty}, \Phi_{d_{2}}\right) \subset \Phi_{d_{2}}$ (ii) follows.

Next we state a standard deformation lemma which is very useful. The proof is left to the reader since it is very simple and follows immediately from Theorem 1.

CoRollary 2. Let $\Phi \in \Sigma$ and suppose there exist numbers $d_{1}, d_{2} \in \mathbf{R}, d_{1} \leqslant d_{2}$, and $\varepsilon>0$ such that $\left\|\Phi^{\prime}(u)\right\| \geqslant \varepsilon$ for all $u \in \Phi_{d_{1}}^{d_{2}}$. Then for an arbitrarily given open neighbourhood $U$ of $\Phi_{d_{1}}^{d_{2}}$ there exists a flow $\eta \in M$, continuously differentiable with respect to $t \in \mathbf{R}$ with

(i) $d \Phi\left(\eta^{t}(u)\right) / d t \geqslant 0$ for all $(t, u) \in \mathbf{R} \times H$.

(ii) $\eta^{1}\left(\Phi_{d_{1}}\right) \subset \Phi_{d_{2}}$.

(iii) $\eta^{t} \mid H \backslash U=$ Id for all $t \in \mathbf{R}$.

Now we are able to define for a given functional $\Phi \in \Sigma$ a set $D_{\Phi}$ of maps $\sigma$ : $H \rightarrow H$, which is useful for defining MM-theories for $\Phi$. If $\Phi$ verifies (WPS), then $D_{\Phi}$ has property $(\mathrm{D})$.

Definition 5. Let $\Phi \in \Sigma$. We denote by $D_{\Phi}$ the set consisting of all maps $\sigma$ : $H \rightarrow H$ verifying $\sigma=\eta^{1}$ for some $\eta \in M$ such that the map $t \rightarrow \Phi\left(\eta^{t}(u)\right)$ is nondecreasing on $[0,1]$.

Proposition 1. The set $D_{\Phi}$ has the following properties:

(i) For all $d \in \mathbf{R}$ the following alternative holds: either there exists a sequence $\left(u_{n}\right) \subset H$ with $\Phi^{\prime}\left(u_{n}\right) \rightarrow 0$ and $\Phi\left(u_{n}\right) \rightarrow d$, or there exist $\varepsilon>0$ and $\sigma \in D_{\Phi}$ with $\sigma\left(\Phi_{d-\varepsilon}\right) \subset \Phi_{d+\varepsilon}$.

(ii) $\boldsymbol{\sigma} \circ \tilde{\boldsymbol{\sigma}} \in D_{\Phi}$ for all $\boldsymbol{\sigma}, \tilde{\boldsymbol{\sigma}} \in D_{\Phi}$.

(iii) All $\sigma \in D$ are homotopic to Id by a map $\eta \in M$ such that $\eta^{0}=\mathrm{Id}, \sigma=\eta^{1}$ and $t \rightarrow \Phi\left(\eta^{t}(u)\right)$ is nondecreasing on $[0,1]$.

REMARK. If $\Phi \in \Sigma$ verifies (WPS) the set $D_{\Phi}$ has property (D).

Proof. (iii) follows immediately from the definition of $D_{\Phi}$. (i) is a consequence of Corollary 2. In order to show (ii) let $\sigma$ and $\tilde{\sigma} \in D$ and denote by $\eta$ and $\tilde{\eta}$ the 
corresponding maps in $M$. Define $\chi \in M$ by

$$
\chi(t, u)= \begin{cases}\tilde{\eta}(2 t, u), & t \leqslant \frac{1}{2} \\ \eta(2 t-1, \tilde{\eta}(1, u)), & t \geqslant \frac{1}{2} .\end{cases}
$$

Then $t \rightarrow \Phi\left(\chi^{t}(u)\right)$ is nondecreasing on $[0,1]$ and $\chi^{1}=\sigma \circ \tilde{\sigma}$. Hence $\sigma \circ \tilde{\sigma} \in M$.

By Proposition 1 we know that for a given functional $\Phi \in \Sigma$ verifying (WPS) there exists a set $D_{\Phi}$ verifying (D) and having some additional useful properties. In order to construct MM-theories for $\Phi$ we have to study the topological properties of maps in $D_{\Phi}$, or more generally the properties of maps in $M$. This will be done in the next section.

2. Topological propertis of elements in $M$ and nontrivial intersections of pairs of sets. In this section we define the notion of a nontrivial intersection of pairs $\left(U_{2}, U_{1}\right)$ and $\left(V_{2}, V_{1}\right)$, where $U_{1} \subset U_{2}$ and $V_{1} \subset V_{2}$ are subsets of $H$. Further we will develop methods in order to give a criterion for such intersections.

Definition 6. Let $U_{1} \subset U_{2}$ and $V_{1} \subset V_{2}$ be subsets of $H$ with $U_{1} \cap V_{2}=\varnothing=U_{2}$ $\cap V_{1}$. We say that $\left(U_{2}, U_{1}\right)$ intersects $\left(V_{2}, V_{1}\right)$ nontrivially with respect to $M$ if we have for all $\eta \in M$ :

$$
\begin{aligned}
& \text { If } \eta^{t}\left(U_{1}\right) \cap V_{2}=\varnothing=\eta^{t}\left(U_{2}\right) \cap V_{1} \quad \text { for all } t \in \mathbf{R} \\
& \text { then } \eta^{t}\left(U_{2}\right) \cap V_{2} \neq \varnothing \quad \text { for all } t \in \mathbf{R} .
\end{aligned}
$$

In that case we write $\left(U_{2}, U_{1}\right) \stackrel{M}{\sim}\left(V_{2}, V_{1}\right)$.

REMARK 1. $\left(U_{2}, U_{1}\right) \stackrel{M}{\sim}\left(V_{2}, V_{1}\right)$ and $\left(V_{2}, V_{1}\right) \stackrel{M}{\sim}\left(U_{2}, U_{1}\right)$ are equivalent statements by the group structure on $M$.

REMARK 2. Assume $J$ is a compact interval in $\mathbf{R}$ containing 0 . Then, if $\left(U_{2}, U_{1}\right) \stackrel{M}{\sim}\left(V_{2}, V_{1}\right)$ and $\eta^{t}\left(U_{1}\right) \cap V_{2}=\varnothing=\eta^{t}\left(U_{2}\right) \cap V_{1}$ for all $t \in J$ we infer that $\eta^{t}\left(U_{2}\right) \cap V_{2} \neq \varnothing$ for all $t \in J$. In fact, we find a continuous map $r: \mathbf{R} \rightarrow J$ such that $r(s)=a$ for $s \leqslant a, r(s)=b$ for $s \geqslant b$, and $r(0)=0$, where $J=[a, b]$. Define $\chi \in M$ by $\chi(t, u)=\eta(r(t), u)$. Our hypotheses imply that $\chi$ verifies $\chi^{t}\left(U_{1}\right) \cap V_{2}=$ $\varnothing=\chi^{t}\left(U_{2}\right) \cap V_{1}$ for all $t \in \mathbf{R}$. Hence $\chi^{t}\left(U_{1}\right) \cap V_{2} \neq \varnothing$ for all $t \in \mathbf{R}$, which implies $\eta^{t}\left(U_{2}\right) \cap V_{2} \neq \varnothing$ for all $t \in J$.

Let $W_{1} \subset W_{2}$ be subsets of $H$. For a given $\eta \in M$ we denote by $\left(W_{2}, W_{1}\right)_{\eta}$ the pair $\left(\eta^{1}\left(W_{2}\right), \eta^{1}\left(W_{1}\right)\right)$. We have the following useful result giving a tool to construct new interesting pairs from known ones.

LemMA 2. If $\left(U_{2}, U_{1}\right) \stackrel{M}{\sim}\left(V_{2}, V_{1}\right)$ then $\left(U_{2}, U_{1}\right)_{\eta} \stackrel{M}{\sim}\left(V_{2}, V_{1}\right)_{\chi}$ for all $\eta, \chi \in M$ such that $\eta^{t}\left(U_{1}\right) \cap \chi^{t}\left(V_{2}\right)=\varnothing=\eta^{t}\left(U_{2}\right) \cap \chi^{t}\left(V_{1}\right)$ for all $t \in[0,1]$.

Proof. Let $\delta \in M$ such that $\delta^{t}\left(\eta^{1}\left(U_{1}\right)\right) \cap \chi^{1}\left(V_{2}\right)=\varnothing=\delta^{t}\left(\eta^{1}\left(U_{2}\right)\right) \cap \chi^{1}\left(V_{1}\right)$ for all $t \in \mathbf{R}$. We have to show that

$$
\delta^{t}\left(\eta^{1}\left(U_{2}\right)\right) \cap \chi^{1}\left(V_{2}\right) \neq \varnothing \quad \text { for all } t \in \mathbf{R} .
$$


Define $\rho \in M$ by

$$
\rho(t, u)= \begin{cases}\chi^{*}(1, \delta(t-1, \eta(1, u))), & t \geqslant 1, \\ \chi^{*}(t, \eta(t, u)), & t \in[0,1], \\ \chi^{*}(-t, \eta(-t, u)), & t \in[-1,0], \\ \chi^{*}(1, \delta(t+1, \eta(1, u))), & t \leqslant-1 .\end{cases}
$$

By our hypothesis

$$
\rho^{t}\left(U_{2}\right) \cap V_{1}=\varnothing=\rho^{t}\left(U_{1}\right) \cap V_{2} \text { for all } t \in \mathbf{R} .
$$

Since $\left(U_{2}, U_{1}\right) \stackrel{M}{\sim}\left(V_{2}, V_{1}\right)$ we infer $\rho^{t}\left(U_{2}\right) \cap V_{2} \neq \varnothing$ for all $t \in \mathbf{R}$. This implies by the definition of $\rho$ the desired result.

Now we will give some examples of nontrivial intersections. The proof of the following statements is an easy application of a theorem which will be stated later. Let $A$ and $Q$ be as before.

Proposition 2. (i) Let $W \subset Q H$ and $W^{\prime} \subset(I-Q) H$ be relatively open bounded sets with $W \cap W^{\prime}=\{0\}$. Define $U_{2}=\operatorname{cl}(W), U_{1}=\partial_{Q H} W, \quad V_{2}=\operatorname{cl}\left(W^{\prime}\right), \quad V_{1}=$

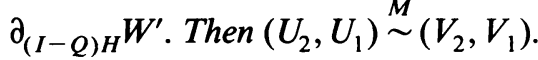

(ii) Let $P=Q$ or $P=I-Q$ and $W \subset P H$ be a relatively open bounded set with $0 \in W$. Define $U_{2}=\operatorname{cl}(W), \quad U_{1}=\partial_{P H} W, \quad V_{2}=(I-P) H, \quad V_{1}=\varnothing$. Then $\left(U_{2}, U_{1}\right) \stackrel{M}{\sim}\left(V_{2}, V_{1}\right)$.

(iii) Let $P=Q$ or $P=I-Q$. Define $U_{2}=\{u \in P H \mid\|u\|=1\}, U_{1}=\varnothing, V_{2}=$ $[0,2] \alpha \oplus\{u \in(I-P) H \mid\|u\| \leqslant 1\}, V_{1}=\partial_{\mathbf{R} \alpha \oplus(I-P) H} V_{2}$, where $\alpha \in P H$ with $\|\alpha\|$ =1. Then $\left(U_{2}, U_{1}\right) \stackrel{M}{\sim}\left(V_{2}, V_{1}\right)$.

(iv) Let $P=Q$ or $P=I-Q$. Define $U_{2}=\{u \in P H \mid\|u\|=1\}, U_{1}=\varnothing, V_{1}=$ $(I-P) H, V_{2}=\mathbf{R}^{+} \alpha \oplus(I-P) H$, $\alpha$ as in (iii). Then $\left(U_{2}, U_{1}\right) \stackrel{M}{\sim}\left(V_{2}, V_{1}\right)$.

In order to prove Proposition 2 we need the following Theorem 2 . We denote by $d(\cdot, \cdot, \cdot)$ the Leray-Schauder degree, with the convention $d(T,\{0\}, 0)=1$ for a map $T:\{0\} \rightarrow\{0\}$.

Let $A \in L(H)$ be selfadjoint.

THEOREM 2. Let $Q_{i}: H \rightarrow H_{i}, i=1,2,3$, be orthogonal projections commuting with $A$ such that $H$ admits the orthogonal decomposition $H=\bigoplus_{i} H_{i}$. Suppose $\operatorname{dim}\left(H_{2}\right)<$ $+\infty$ and $U \subset H_{1} \oplus H_{2}$ and $V \subset H_{2} \oplus H_{3}$ are relatively open bounded sets with $U \cap V \neq \varnothing$. Assume $\delta:\left(H_{1} \oplus H_{2}\right) \times\left(H_{2} \oplus H_{3}\right) \rightarrow H_{2}$ is a continuous bounded map, $\eta \in M=M\left(H, A, Q_{1}+Q_{2}\right)$, and $J$ a compact interval containing $0 \in \mathbf{R}$ such that

$$
\eta^{t}(u)-v \neq 0 \text { for all }(t, u, v) \in J \times\left(\partial(U \times V) \cap \delta^{-1}(0)\right) .
$$

Define $\delta^{*}: H_{2} \rightarrow H_{2}$ by $\delta^{*}(u)=\delta(u, u)$ and suppose $d\left(\delta^{*}, U \cap V, 0\right) \neq 0$. Then the problem $\eta^{t}(u)=v, \delta(u, v)=0,(u, v) \in U \times V$, is solvable for all $t \in J$.

RemarK. Note that $d\left(\delta^{*}, U \cap V, 0\right)$ is well defined if $\operatorname{dim}\left(H_{2}\right) \geqslant 1$ since in that case $\delta^{*}(u) \neq 0$ for $u \in \partial(U \cap V)$. 
Proof. Let $\eta(t, u)=\gamma(t, u) u+C(t, u)$, where the decomposition is as in (1). Clearly

(7)

$$
\eta(t, u)-v=0, \quad \delta(u, v)=0 \quad \text { for some }(t, u, v) \in \Gamma=J \times \operatorname{cl}(U) \times \operatorname{cl}(V)
$$

is equivalent to

$$
\begin{aligned}
0 & =u+(\gamma(t, u))^{-1}\left(Q_{1}+Q_{2}\right)\left(C(t, u)-Q_{2} v+\delta(u, v)\right) \\
& =: u+C^{*}(t, u, v), \\
0 & =v-\gamma(t, u) Q_{2} u-\left(Q_{2}+Q_{3}\right) C(t, u)+\delta(u, v) \\
& =: v+C^{* *}(t, u, v)
\end{aligned}
$$

for some $(t, u, v) \in \Gamma$.

Note that $\left(Q_{2}+Q_{3}\right) C$ is not compact and therefore $C^{* *}$ is not compact. Since $C^{*}: \Gamma \rightarrow H$ is compact and $\Gamma$ bounded we find that $C^{*} \Gamma$ is relatively compact. Therefore there exists a compact subset $\Omega^{\prime} \subset \operatorname{cl}(U)$ such that $u \in \dot{\Omega}^{\prime}$ for all $(t, u, v)$ $\in \Gamma$ being a solution of $u+C^{*}(t, u, v)=0$. Let

$$
\Omega=\left(J \times \Omega^{\prime}\right) \cup(\{0\} \times \operatorname{cl}(U)) .
$$

Observe that $\left(Q_{2}+Q_{3}\right) C \mid \Omega$ is compact since $J \times \Omega^{\prime}$ is compact and $C \mid\{0\} \times$ $\operatorname{cl}(U)=0$. By a well-known extension theorem there exists a compact extension $C^{\prime}$ : $J \times \operatorname{cl}(U) \rightarrow H$ of $\left(Q_{2}+Q_{3}\right) C \mid \Omega$. Hence the operator $C^{\prime \prime}: \Gamma \rightarrow H$ defined by

$$
C^{\prime \prime}(t, u, v)=-\gamma(t, u) Q_{2} u-\left(Q_{2}+Q_{3}\right) C^{\prime}(t, u)+\delta(u, v)
$$

is compact. Define an operator $T: \Gamma \rightarrow\left(H_{1} \oplus H_{2}\right) \times\left(H_{2} \oplus H_{3}\right)$ by

$$
T(t, u, v)=\left(u+C^{*}(t, u, v), v+C^{\prime \prime}(t, u, v)\right) .
$$

Obviously the solutions $(t, u, v) \in \Gamma$ of $T(t, u, v)=0$ are exactly the solutions of (7). Moreover $T(0, u, v)=\left(u-Q_{2} v+\delta(u, v), v-Q_{2} u+\delta(u, v)\right)$. By our hypotheses we have $T(t, u, v) \neq 0$ for all $(t, u, v) \in J \times \partial(U \times V)$. By the homotopy invariance of the degree

$$
d(t):=d(T(t, \cdot), U \times V, 0)=d(T(0, \cdot), U \times V, 0)=d(0) .
$$

Define a homotopy $T^{1}:[0,1] \times\left(H_{1} \oplus H_{2}\right) \times\left(H_{2} \oplus H_{3}\right) \rightarrow\left(H_{1} \oplus H_{2}\right) \times\left(H_{2} \oplus\right.$ $\mathrm{H}_{3}$ ) by

$$
\begin{array}{r}
T^{1}(t, u, v)=\left(Q_{1} u+t Q_{2}(u-v)+\delta\left(t Q_{1} u+Q_{2} u, t v+(1-t) Q_{2} u\right),\right. \\
\left.v-Q_{2} u+t \delta\left(t Q_{1} u+Q_{2} u, t v+(1-t) Q_{2} u\right)\right) .
\end{array}
$$

Clearly $T^{\mathrm{l}}(1, u, v)=T(0, u, v)$ for $(u, v) \in \operatorname{cl}(U) \times \operatorname{cl}(V)$ and $T^{1}(0, u, v)=$ $\left(Q_{1} u+\delta^{*}\left(Q_{2} u\right), v-Q_{2} u\right)$. Assume $T^{1}(t, u, v)=0$ for some $(t, u, v) \in[0,1] \times$ $\partial(U \times V)$. We deduce $Q_{1} u=0=Q_{3} v$, such that $u=Q_{2} u$ and $v=Q_{2} v$. Hence we must have

$$
\begin{aligned}
& t(u-v)+\delta(u, t v+(1-t) u)=0 \\
& (v-u)+t \delta(u, t v+(1-t) u)=0 .
\end{aligned}
$$

This implies $\left(1+t^{2}\right)(v-u)=0$. Therefore $u=v$ and $\delta^{*}(u)=0$. Now $(u, u)=$ $(u, v) \in(\partial(U \times V)) \cap\left(H_{2} \times H_{2}\right)$ and we find that $u \in \partial_{H_{2}}(U \cap V)$, which implies 
a contradiction. By the homotopy invariance of the degree

$$
d(t)=d\left(T^{1}(0, \cdot), U \times V, 0\right) .
$$

Define a set $\tilde{\Gamma} \subset\left(H_{1} \oplus H_{2}\right) \times\left(H_{2} \oplus H_{3}\right)$ by

$$
\tilde{\Gamma}=\left(Q_{1} U \oplus(U \cap V)\right) \times\left((U \cap V) \oplus Q_{3} V\right) .
$$

If $T^{\mathrm{l}}(0, u, v)=0$ for some $(u, v) \in \operatorname{cl}(\tilde{\Gamma})$ we infer that $(u, v) \in(U \cap V) \times$ $(U \cap V)$. The same conclusion holds by the preceding discussion if we assume $T^{1}(0, u, v)=0$. for some $(u, v) \in \operatorname{cl}(U) \times \operatorname{cl}(V)$. By the excision property

$$
\begin{aligned}
d(t) & =d\left(T^{\mathrm{l}}(0, \cdot), \tilde{\Gamma}, 0\right) \\
& =d\left(\mathrm{Id}, Q_{1} U \times Q_{3} V, 0\right) d\left(T^{2}, D \times D, 0\right) \\
& =d\left(T^{2}, D \times D, 0\right)
\end{aligned}
$$

since $(0,0) \in Q_{1} U \times Q_{3} V$, where $D=U \cap V$ and $T^{2}: H_{2} \times H_{2} \rightarrow H_{2} \times H_{2}$ is defined by

$$
T^{2}(u, v)=\left(\delta^{*}(u), v-u\right) .
$$

Denote by $D^{*}$ an open ball in $H_{2}$ containing $0 \in H_{2}$ and $\operatorname{cl}(D)$. By the excision property

$$
d(t)=d\left(T^{2}, D \times D^{*}, 0\right) .
$$

Define a homotopy $T^{3}:[0,1] \times \operatorname{cl}(D) \times \operatorname{cl}\left(D^{*}\right) \rightarrow H_{2} \times H_{2}$ by

$$
T^{3}(t, u, v)=\left(\delta^{*}(u), v-t u\right) .
$$

For all $(t, u, v) \in[0,1] \times \partial\left(D \times D^{*}\right)$ we infer, since $\operatorname{cl}(D) \subset D^{*}, T^{3}(t, u, v) \neq 0$. Moreover $T^{3}(1, \cdot)=T^{2}$ and $T^{3}(0, \cdot)=\delta^{*} \times$ Id. Hence

$$
\begin{aligned}
d(t) & =d\left(\delta^{*} \times \operatorname{Id}, D \times D^{*}, 0\right)=d\left(\delta^{*}, D, 0\right) d\left(\mathrm{Id}, D^{*}, 0\right) \\
& =d\left(\delta^{*}, D, 0\right) \neq 0 .
\end{aligned}
$$

This proves the theorem.

Proof of Proposition 2. (i) Let $Q_{1}=Q, Q_{2}=0, Q_{3}=\mathrm{Id}-Q$, and $\delta=0$. Define $U=W$ and $V=W^{\prime}$. Then $U \cap V=\{0\}$ and $d\left(\delta^{*},\{0\}, 0\right)=1$. Let $\eta \in$ $M(H, A, Q)=M\left(H, A, Q_{1}+Q_{2}\right)$ such that

$$
\eta^{t}\left(U_{2}\right) \cap V_{1}=\varnothing=\eta^{t}\left(U_{1}\right) \cap V_{2} \text { for all } t \in \mathbf{R} \text {. }
$$

This is equivalent to

$$
\eta^{t}(u)-v \neq 0 \text { for all }(t, u, v) \in \mathbf{R} \times \partial(U \times V) .
$$

An application of Theorem 2 yields the desired result.

(ii) Let $P=\mathrm{Id}-Q$ and define $Q_{1}=Q, Q_{2}=0, Q_{3}=\mathrm{Id}-Q$. Let $\eta \in$ $M(H, A, Q)$ such that

$$
\eta^{t}\left(U_{2}\right) \cap V_{1}=\varnothing=\eta^{t}\left(U_{1}\right) \cap V_{2} \quad \text { for all } t \in \mathbf{R} .
$$

This is equivalent to

$$
\chi^{t}\left(V_{1}\right) \cap U_{2}=\varnothing=\chi^{t}\left(V_{2}\right) \cap U_{1} \text { for all } t \in \mathbf{R},
$$


where $\chi=\eta^{*}$. We have to show that $\chi^{t}\left(V_{2}\right) \cap U_{2} \neq \varnothing$ for all $t \in \mathbf{R}$. Fix any $\hat{t} \in \mathbf{R}$ and define $J=[\min \{0, \hat{t}\}, \max \{0, \hat{t}\}]$. Since $\eta$ is of class $C_{b}$ we find numbers $r>0$ and $d>0$ such that

$$
\left\|\chi^{t}(u)\right\| \geqslant d \quad \text { for all } u \in V_{2} \backslash B_{r} \quad \text { and } \quad d>\sup _{u \in U_{2}}\|u\| \quad \text { for all } t \in J .
$$

Define $U=V_{2} \cap B_{r}$ and $V=W$. By our hypotheses

$$
\chi^{t}(u)-v \neq 0 \text { for all }(t, u, v) \in J \times \partial(U \times V) .
$$

An application of Theorem 2 yields the desired result. The somewhat easier case $P=Q$ is left to the reader.

(iii) Let $P=\mathrm{Id}-Q$. Deffne $V=\{u \in(\mathrm{Id}-Q) H \mid\|u\|<2\}, \quad U=(0,2) \alpha \oplus$ $\{u \in Q H \mid\|u\|<1\}, H_{1}=Q H, H_{2}=\mathbf{R} \alpha, H_{3}=(\operatorname{Id}-Q) H \cap\{\alpha\}^{\perp}$, and $\delta(u, v)$ $=(\|v\|-1) \alpha$. Now let $\eta \in M$ with

$$
\eta^{t}\left(U_{2}\right) \cap V_{1}=\varnothing=\eta^{t}\left(U_{1}\right) \cap V_{2} \text { for all } t \in \mathbf{R} .
$$

Observe that $\chi=\eta^{*} \in M$ and that the preceding is equivalent to

$$
\chi^{t}(u)-v \neq 0 \text { for all }(t, u, v) \in \mathbf{R} \times\left(\partial(U \times V) \cap \delta^{-1}(0)\right) .
$$

Since $d(\delta, U \cap V, 0)=d(\mathrm{Id},(0,2) \alpha, \alpha)=1$ an application of Theorem 2 yields the desired result. The case $P=Q$ is left to the reader.

(iv) The proof is a combination of (ii) and (iii) and is left to the reader.

3. Existence results for critical points. Combining results from $\S 1$ and $\S 2$ we will prove an existence and a perturbation result. First introduce a subset $\Sigma_{C}$ of $\Sigma$ by

Definition 7. Denote by $\Sigma_{C}$ the subset of $\Sigma$ consisting of all $\Phi \in \Sigma$ verifying (WPS).

We have

THEOREM 3. Let $\Phi \in \Sigma_{C}$ and assume $\left(U_{2}, U_{1}\right) \stackrel{M}{\sim}\left(V_{2}, V_{1}\right)$ such that

$$
\inf \Phi\left(U_{2}\right)>\sup \Phi\left(V_{1}\right) \text { and } \inf \Phi\left(U_{1}\right)>\sup \Phi\left(V_{2}\right) \text {. }
$$

Then $\left(D_{\Phi}, S, c\right)$, where $S=\left\{\sigma\left(U_{2}\right) \mid \sigma \in D_{\Phi}\right\}$ and $c=\sup _{\xi \in S} \inf \Phi(\xi)$, is a MM-theory. Hence $c$ is a critical value. Moreover $c \in\left[\inf \Phi\left(U_{2}\right)\right.$, sup $\left.\Phi\left(V_{2}\right)\right]$.

Proof. By Proposition 1, $D_{\Phi}$ verifies (D) since $\Phi \in \Sigma_{C}$, and $D_{\Phi}(S) \subset S$. It remains to show that $c \in\left[\inf \Phi\left(U_{2}\right), \sup \Phi\left(V_{2}\right)\right] \subset \mathbf{R}$. It is enough to prove that $\sigma\left(U_{2}\right) \cap V_{2} \neq \varnothing$ for all $\sigma \in D_{\Phi}$. By the definition of $D_{\Phi}$ there exists $\eta \in M$ such that the map $t \rightarrow \Phi\left(\eta^{t}(u)\right)$ is nondecreasing on $[0,1]$ and $\sigma=\eta^{1}$. By (8) we infer that

$$
\eta^{t}\left(U_{2}\right) \cap V_{1}=\varnothing=\eta^{t}\left(U_{1}\right) \cap V_{2} \text { for all } t \in[0,1] .
$$

Hence $\left(U_{2}, U_{1}\right)_{\eta} \stackrel{M}{\sim}\left(V_{2}, V_{1}\right)$ by Lemma 2 , which implies $\sigma\left(U_{2}\right) \cap V_{2} \neq \varnothing$. Therefore

$$
\inf \Phi\left(U_{2}\right) \leqslant c \leqslant \sup \Phi\left(V_{2}\right) \text {. }
$$

By the preceding discussion $\left(D_{\Phi}, S, c\right)$ is a MM-theory and by Theorem (MMP), $c$ is a critical value.

Theorem 3 together with Proposition 2 gives a useful tool in studying the critical points of a functional $\Phi \in \Sigma$. This will be illustrated in the applications. 
Next we shall prove a perturbation result. For this we introduce a subset $\Sigma_{*}$ of $\Sigma$ containing $\Sigma_{C}$.

Definition 8. Denote by $\Sigma_{*}$ the subset of $\Sigma$ consisting of all $\Phi$ verifying

(*) If $\Phi^{\prime}\left(u_{n}\right) \rightarrow 0$ and $\Phi\left(u_{n}\right) \rightarrow d \in \mathbf{R}$ for some bounded sequence $\left(u_{n}\right)$, then $d$ is a critical value of $\Phi$.

It is useful to equip $\Sigma$ with a topology $T_{\Sigma}$. Let $T_{\Sigma}$ be the topology generated by the subbasis consisting of all sets of the form

$$
V(\Phi, C, \varepsilon)=\left\{\Psi \in \Sigma \mid \sup _{u \in C}\left(|\Psi(u)-\Phi(u)|+\left\|\Psi^{\prime}(u)-\Phi^{\prime}(u)\right\|\right)<\varepsilon\right\},
$$

where $\Phi \in \Sigma, C$ is a bounded subset of $H$, and $\varepsilon>0$. We have

THEOREM 4. Let $\Phi \in \Sigma$ and assume $\left(U_{2}, U_{1}\right) \stackrel{M}{\sim}\left(V_{2}, V_{1}\right)$ where $U_{2}$ and $V_{2}$ are bounded sets such that

$$
\inf \Phi\left(U_{2}\right)>\sup \Phi\left(V_{1}\right) \text { and } \inf \Phi\left(U_{1}\right)>\sup \Phi\left(V_{2}\right) \text {. }
$$

Let $\tilde{\varepsilon}>0$ be arbitrarily given and define $d_{1}=\inf \Phi\left(U_{2}\right)-\tilde{\varepsilon}, d_{2}=\sup \Phi\left(V_{2}\right)+\tilde{\varepsilon}$. Then there exists a neighbourhood $W \in T_{\Sigma}$ of $\Phi$ such that

(i) for given $\Psi \in W$ there exists a sequence $\left(u_{n}\right)$ such that $\Psi^{\prime}\left(u_{n}\right) \rightarrow 0$ and $\Psi\left(u_{n}\right) \rightarrow d \in\left[d_{1}, d_{2}\right]$ for some $d$,

(ii) if in addition $\Phi \in \Sigma_{C}$ there exists for all $\Psi \in \Sigma_{*} \cap W$ a critical point $u \in \Psi_{d_{1}}^{d_{2}}$.

Proof. We carry out the proof only for (ii). The proof of (i) is essentially simpler and is left to the reader.

Let $\Phi \in \Sigma_{C}$. By the (WPS)-condition we find $\varepsilon>0$ and $s>0$ such that

$$
\left\|\Phi^{\prime}(u)\right\| \geqslant \varepsilon \quad \text { for all } u \in \Phi_{d_{1}-\tilde{\varepsilon}}^{d_{2}+\tilde{\varepsilon}} \backslash B_{s} .
$$

By increasing $s$ if necessary we may assume $\operatorname{cl}\left(U_{2}\right) \subset B_{s}$. Choose a number $r \in \mathbf{R}$ such that

$$
\ln (r /(s+1)) \geqslant 2\left(d_{2}-d_{1}\right) / \alpha \quad \text { with } \alpha=\varepsilon / 2 .
$$

Observe that $d_{2}-d_{1} \geqslant 2 \tilde{\varepsilon}$ since $U_{2} \cap V_{2} \neq \varnothing$. Using that $U_{2}$ and $V_{2}$ are bounded sets we find a neighbourhood $W \in T_{\Sigma}$ of $\Phi$ such that

$$
\begin{gathered}
d_{1}<\inf \Psi\left(U_{2}\right) \leqslant \sup \Psi\left(V_{2}\right)<d_{2}, \\
\inf \Psi\left(U_{2}\right)>\sup \Psi\left(V_{1}\right), \quad \inf \Psi\left(U_{1}\right)>\sup \Psi\left(V_{2}\right), \\
\left\|\Psi^{\prime}(u)\right\| \geqslant \alpha \quad \text { for } u \in \Psi_{d_{1}}^{d_{2}} \cap\left(B_{r} \backslash B_{s}\right),
\end{gathered}
$$

for all $\Psi \in W$. Now let $\Psi \in W \cap \Sigma_{*}$. Define

$$
a:=\inf \left\{\left\|\Psi^{\prime}(u)\right\| \mid u \in B_{r} \cap \Psi_{d_{1}}^{d_{2}}\right\} .
$$

If $a=0$ we are done by (*). Hence we may assume $a>0$. By Corollary 1 we find in that case $\eta \in M$ such that

$$
\begin{gathered}
\frac{d}{d t} \Psi\left(\eta^{t}(u)\right) \geqslant 0 \text { for all } t, u, \\
\eta^{t_{\infty}}\left(B_{s} \cap \Psi_{d_{1}}\right) \subset \Psi_{d_{2}} \text { for some } t_{\infty} \text { large enough. }
\end{gathered}
$$


By (10), since $\left(U_{2}, U_{1}\right) \stackrel{M}{\sim}\left(V_{2}, V_{1}\right)$, we deduce

$$
\eta^{t}\left(U_{2}\right) \cap V_{2} \neq \varnothing \quad \text { for all } t \in \mathbf{R}^{+} .
$$

Observing that $U_{2} \subset B_{s} \cap \Psi_{d_{1}}$ we deduce $\eta^{t_{\infty}}\left(U_{2}\right) \subset \Psi_{d_{2}}$, which implies $\eta^{t_{\infty}}\left(U_{2}\right) \cap V_{2}$ $=\varnothing$ since $V_{2} \subset \operatorname{int}\left(\Psi^{d_{2}}\right)$. This contradiction proves (ii).

\section{APPLICATIONS TO LANDESMAN-LAZER-TYPE PROBLEMS}

1. Abstract results. Let $(H,(\cdot, \cdot))$ be a real Hilbert space and $\Phi \in C^{1}(H, \mathbf{R})$. We impose the following hypotheses on $\Phi$ :

( $\Phi)$ The functional $\Phi \in C^{1}(H, \mathbf{R})$ has a gradient $\Phi^{\prime}$ admitting the decomposition $\Phi^{\prime}=A+B$, where $A \in L(H)$ is selfadjoint and $B \in C(H, H)$ with bounded range. Denote by $b$ the potential of $B$, normalized by $b(0)=0$. Suppose $H$ admits the orthogonal decomposition $H=X \oplus Y \oplus Z$, with $X, Y$, and $Z$ being $A$-invariant closed subspaces having corresponding orthogonal projections $P_{-}: H \rightarrow X, P$ : $H \rightarrow Y$, and $P_{+}: H \rightarrow Z$, such that $1 \leqslant \operatorname{dim}(Y)<+\infty$. Moreover assume that the potential $a: H \rightarrow \mathbf{R}$ of $A, a(u)=\frac{1}{2}(A u, u)$, verifies (with $\alpha>0$ )

$$
\begin{array}{ll}
a(x) \geqslant \alpha\|x\|^{2}, & x \in X, \\
a(y)=0, & y \in Y, \\
a(z) \leqslant-\alpha\|z\|^{2}, & z \in Z .
\end{array}
$$

Let $Q=P_{+}$and suppose that $Q B$ is compact. Abbreviate $\Sigma(H, A, Q)$ and $M(H, A, Q)$ by $\Sigma$ and $M$. Observe that $\Phi \in \Sigma$ and assume in addition that $\Phi_{h} \in \Sigma_{*}$ for all $h \in H$, where $\Phi_{h}=\Phi+(h, \cdot)$.

We define a set $\theta \subset H$ by

$$
\begin{aligned}
\theta=\{h \in H \mid & \lim \left(b\left(y_{n}\right)+\left(h, y_{n}\right)\right)=+\infty \\
& \text { for all sequences } \left.\left(y_{n}\right) \subset y,\left\|y_{n}\right\| \rightarrow+\infty\right\},
\end{aligned}
$$

and a positively homogeneous functional $J_{b}: Y \rightarrow \mathbf{R}$ by

$$
J_{b}(u)=\liminf _{t \rightarrow+\infty} b(t y) / t .
$$

Then $J_{b}$ is Lipschitz continuous with constant $\|B\|=\sup \{\|B u\| \mid u \in H\}$. In fact, $b(t y) / t \leqslant b\left(t y^{\prime}\right) / t+\|B\|\left\|y-y^{\prime}\right\|$, from which we infer $J_{b}(y) \leqslant J_{b}\left(y^{\prime}\right)+$ $\|B\|\left\|y-y^{\prime}\right\|$ for all $y, y^{\prime} \in Y$. Now we characterize the set $\theta$ using $J_{b}$ :

LEMMA 3. (i) $\theta$ is convex.

(ii) $\operatorname{int}(\theta)=\left\{h \in H \mid-(h, y)<J_{b}(y)\right.$ for all $\left.y \in Y \backslash\{0\}\right\}$.

(iii) $\operatorname{cl}(\theta) \subset\left\{h \in H \mid-(h, y) \leqslant J_{b}(y)\right.$ for all $\left.y \in Y\right\}$.

(iv) If for all sequences $\left(y_{n}\right) \subset Y,\left\|y_{n}\right\| \rightarrow+\infty$, we have $\lim \left(b\left(y_{n}\right)-J_{b}\left(y_{n}\right)\right)=$ $+\infty$, then $\theta$ is closed and given by $\theta=\left\{h \in H \mid-(h, y) \leqslant J_{b}(y)\right.$ for all $\left.y \in Y\right\}$.

The proof will be given later. We want to remark that $J_{b}$ is related to the recession function introduced by Brézis and Nirenberg [9]. However, there is an important difference: instead of using the asymptotic behaviour of $B$ we make use of the behaviour of $b(u) /\|u\|$. In general, our function $J_{b}$ gives more information than the recession function of Brézis and Nirenberg. The reader should note that the recession function is also defined in the nonvariational case. 
Our first result is the following:

TheOrem 5. Assume $\Phi$ verifies $(\Phi)$. Suppose that for all sequences $\left(u_{n}\right) \subset H$ with ((Id $\left.-P) u_{n}\right)$ bounded and $\left\|P u_{n}\right\| \rightarrow+\infty$ the following holds:

$$
\begin{aligned}
\lim \left(b\left(u_{n}\right)-J_{b}\left(P u_{n}\right)\right) & =+\infty, \\
\lim \left(b\left(u_{n}\right)-\left(B u_{n}, u_{n}\right)\right) & =+\infty .
\end{aligned}
$$

(Then $\theta$ is closed by Lemma 3(iv).)

Then there exists an open set $U$ containing $\theta$ such that

(i) For all $h \dot{\in} U, \Phi_{h}$ possesses at least one critical point.

(ii) For all $h \in U \backslash \theta, \Phi_{h}$ possesses at least two critical points.

Proof. Sketch of the proof. For $h^{*} \in \theta$ we first construct a pair of intersecting sets and show that $\Phi_{h^{*}}$ verifies (WPS). The perturbation result Theorem 4(ii) then yields a first solution, also for $h$ in a neighbourhood $V\left(h^{*}\right)$ of $h^{*}$. For fixed $h \in V\left(h^{*}\right) \backslash \theta$ we construct a second pair of intersecting sets. We cannot prove that $\Phi_{h}$ satisfies (WPS). However this condition holds for very special perturbations of $\Phi_{h}$, which allow passage to the limit.

Step 1. For given $h^{*} \in \theta$ there exists $\delta>0$ such that $U_{1}=\{u \in(I-Q) H \mid\|u\|$ $=\delta\}$ verifies

$$
\inf \Phi_{h^{*}}\left(U_{1}\right)>\sup \Phi_{h^{*}}(Z)
$$

In fact, we have for $z \in Z=Q H$

$$
\begin{aligned}
\Phi_{h^{*}}(z) & =a(z)+b(z)+\left(h^{*}, z\right) \\
& \leqslant-\alpha\|z\|^{2}+\left(\|B\|+\left\|h^{*}\right\|\right)\|z\|,
\end{aligned}
$$

which implies $\sup \Phi_{h}(Z)<+\infty$. On the other hand we estimate, for $u=x+y \in$ $($ Id $-Q) H=X \oplus Y$,

$$
\begin{aligned}
\Phi_{h^{*}}(u) & =a(x)+b(u)+\left(h^{*}, u\right) \\
& \geqslant \alpha\|x\|^{2}+b(y)+\int_{0}^{1}(B(y+t x), x) d t+\left(h^{*}, u\right) \\
& \geqslant \alpha\|x\|^{2}-\left(\|B\|+\left\|h^{*}\right\|\right)\|x\|+b(y)+\left(h^{*}, y\right) .
\end{aligned}
$$

Hence for $\|u\|$ large enough we have $\Phi_{h^{*}}(u)>\sup \Phi_{h^{*}}(Z)$, since $h^{*} \in \theta$. Therefore we find $\delta>0$ having the desired properties.

Step 2. Let $h^{*} \in \boldsymbol{\theta}$. The $\Phi_{h^{*}}$ verifies (WPS).

Assume $\Phi_{h^{*}}\left(u_{n}\right) \rightarrow d$ and $\Phi_{h^{*}}^{\prime}\left(u_{n}\right) \rightarrow 0$. We immediately find that $\left((\operatorname{Id}-P) u_{n}\right)$ is bounded. Hence, if $\left\|u_{n}\right\| \rightarrow+\infty$ we must have $\left\|P u_{n}\right\| \rightarrow+\infty$. We obtain, observing that $h^{*} \in \theta$, since $a\left(u_{n}\right)=a\left((\operatorname{Id}-P) u_{n}\right)$ is bounded,

$$
\begin{aligned}
+\infty & >\limsup \Phi_{h^{*}}\left(u_{n}\right) \\
& \geqslant \text { const }+\lim \left(b\left(P u_{n}\right)+\left(h^{*}, P u_{n}\right)\right) \\
& =+\infty .
\end{aligned}
$$

This contradiction shows that $\left(u_{n}\right)$ must be bounded. Since $\Phi_{h^{*}} \in \Sigma_{*}$, we conclude that $d$ is a critical level. 
Step 3. Assume $h^{*} \in \theta$ is given and $U_{1}$ is as in Step 1. Let $U_{2}=\{u \in(\operatorname{Id}-Q) H \mid$ $\|u\| \leqslant \delta\}$ and $\tilde{\varepsilon}=\frac{1}{4}\left(\inf \Phi_{h^{*}}\left(U_{1}\right)-\sup \Phi_{h^{*}}(Z)\right)(\tilde{\varepsilon}>0)$. Then there exists an open neighbourhood $V\left(h^{*}\right)$ such that $\Phi_{h}$ possesses for all $h \in V\left(h^{*}\right)$ a critical point $u \in\left(\Phi_{h}\right)_{d_{1}}^{d_{2}}$, where $d_{1}=\inf \Phi_{h^{*}}\left(U_{2}\right)-\tilde{\varepsilon}, d_{2}=\sup \Phi_{h^{*}}(Z)+\tilde{\varepsilon}$. Moreover, inf $\Phi_{h}\left(U_{1}\right)$ $\geqslant d_{2}+2 \tilde{\varepsilon}$ and $\sup \Phi_{h}(Z) \leqslant d_{2}$ for all $h \in V\left(h^{*}\right)$. We find $\tau>0$ such that $V_{1}=$ $\{u \in Z \mid\|u\|=\tau\}$ verifies

$$
\sup \Phi_{h^{*}}\left(V_{1}\right)<\inf \Phi_{h^{*}}\left(U_{2}\right)
$$

Define

$$
V_{2}=\{u \in Z \mid\|u\| \leqslant \tau\} .
$$

By Proposition 2(ii) we have $\left(U_{2}, U_{1}\right) \stackrel{M}{\sim}\left(V_{2}, V_{1}\right)$. Moreover,

$$
\begin{aligned}
& \inf \Phi_{h^{*}}\left(U_{2}\right)>\sup \Phi_{h^{*}}\left(V_{1}\right), \\
& \inf \Phi_{h^{*}}\left(U_{1}\right)>\sup \Phi_{h^{*}}\left(V_{2}\right) .
\end{aligned}
$$

By Theorem 4(ii), since $\Phi_{h^{*}}$ verifies (WPS) and $\Phi_{h} \in \Sigma_{*}$ for all $h \in H$, we find a neighbourhood $\hat{V}\left(\mathrm{~h}^{*}\right)$ such that $\Phi_{h}$ possesses a critical point $u \in\left(\Phi_{h}\right)_{d_{1}}^{d_{2}}$ for all $h \in \tilde{V}\left(h^{*}\right)$. By the definition of $\tilde{\varepsilon}$ we have

$$
\inf \Phi_{h^{*}}\left(U_{1}\right)=\sup \Phi_{h^{*}}(Z)+4 \tilde{\varepsilon}=d_{2}+4 \tilde{\varepsilon} .
$$

Replacing eventually $\hat{V}\left(h^{*}\right)$ by a smaller open neighbourhood $V\left(h^{*}\right)$ we may assume for all $h \in V\left(h^{*}\right)$

$$
\begin{gathered}
\inf \Phi_{h}\left(U_{1}\right) \geqslant d_{2}+2 \tilde{\varepsilon}, \\
\sup \Phi_{h}\left(V_{2}\right) \leqslant d_{2} \text { and } \sup \Phi_{h}(Z) \leqslant d_{2} .
\end{gathered}
$$

Observe that the last inequality holds since we can find a constant $r>0$ such that $\sup \Phi_{h}(Z)=\sup \Phi_{h}\left(Z \cap B_{r}\right)$ for all $h \in H$ with $\left\|h-h^{*}\right\| \leqslant 1$. By construction $V\left(h^{*}\right)$ has the desired properties.

Step 4. Let $U=\cup_{h^{*} \in \theta} V\left(h^{*}\right)$, where $V\left(h^{*}\right)$ is the set constructed in Step 3. If $h \in U \backslash \theta$ the $\Phi_{h}$ possesses at least two critical points.

By construction of $U$ we have for all $h \in U$ at least one critical point. If $h \in U \backslash \theta$ there exists $h^{*} \in \theta$ with $h \in V\left(h^{*}\right) \backslash \theta$. Let $U_{1}, d_{2}$ and $\tilde{\varepsilon}>0$ be as in Step 3. We have

$$
\sup \Phi_{h}(Z) \leqslant d_{2}, \quad \inf \Phi_{h}\left(U_{1}\right) \geqslant d_{2}+2 \tilde{\varepsilon}
$$

and $\Phi_{h}$ possesses a critical point $u^{\prime}$ with $\Phi_{h}\left(u^{\prime}\right) \leqslant d_{2}$. Now we will show that $\Phi_{h}$ possesses a critical point $u^{\prime \prime}$ with $\Phi_{h}\left(u^{\prime \prime}\right) \geqslant d_{2}+\tilde{\varepsilon}$, which will complete the proof. By the characterization of $\theta$ given in Lemma 3 we find $y_{0} \in Y,\left\|y_{0}\right\|=1$, with $J_{b}\left(y_{0}\right)<-\left(h, y_{0}\right)$, or equivalently $\liminf _{t \rightarrow+\infty}\left(b\left(t y_{0}\right)+\left(h, t y_{0}\right)\right)=-\infty$. We have for $u=z+t y_{0}, z \in Z, t \geqslant 0$ :

$$
\begin{aligned}
\Phi_{h}(u) & \leqslant-\alpha\|z\|^{2}+(\|B\|+\|h\|)\|z\|+b\left(t y_{0}\right)+\left(h, t y_{0}\right) \\
& \leqslant c_{2}+b\left(t y_{0}\right)+\left(h, t y_{0}\right),
\end{aligned}
$$

where $c_{2}$ is a constant independent of $t \in \mathbf{R}^{+}$and $z \in Z$. Moreover $\sup \Phi_{h}(Z) \leqslant d_{2}$. We find a constant $t_{0}>\delta$ (where $\delta$ is the constant defining $U_{1}$ and $U_{2}$ ) such that

$$
b\left(t_{0} y_{0}\right)+\left(h, t_{0} y_{0}\right) \leqslant d_{2}-c_{2} \text {. }
$$


Let $c_{3}=\sup \left\{b\left(t y_{0}\right)+\left(h, t y_{0}\right) \mid t \in\left[0, t_{0}\right]\right\}$. Since $-\alpha\|z\|^{2}+(\|B\|+\|h\|)\|z\| \leqslant d_{2}$ $-c_{3}$ for all $z \in Z,\|z\| \geqslant \rho_{1}$, for a suitable constant $\rho_{1}>0$, we deduce

$$
\sup \Phi_{h}(\partial V) \leqslant d_{2},
$$

where $V=\left[0, t_{0}\right] y_{0} \oplus\left\{z \in Z \mid\|z\| \leqslant \rho_{0}\right\}$ and $\partial$ denotes the boundary relative to $\mathbf{R} y_{0} \oplus Z$. Define

$$
\begin{array}{ll}
U_{2}^{\prime}=U_{1}, & U_{1}^{\prime}=\varnothing, \\
V_{2}^{\prime}=V, & V_{1}^{\prime}=\partial V .
\end{array}
$$

Then $\left(U_{2}^{\prime}, U_{1}^{\prime}\right) \stackrel{M}{\sim}\left(V_{2}^{\prime}, V_{1}^{\prime}\right)$ by Proposition 2(iii) and moreover,

$$
\begin{aligned}
& \inf \Phi_{h}\left(U_{2}^{\prime}\right)>\sup \Phi_{h}\left(V_{1}^{\prime}\right), \\
& \inf \Phi_{h}\left(U_{1}^{\prime}\right)>\sup \Phi_{h}\left(V_{2}^{\prime}\right) .
\end{aligned}
$$

Applying Theorem 4(i) we find $\varepsilon_{0}>0$ such that for all functionals $\Psi_{\varepsilon}:=\Phi_{h}$ $-\frac{\varepsilon}{2}\|P(\cdot)\|^{2}, \varepsilon \in\left(0, \varepsilon_{0}\right]$, there exists a sequence $\left(u_{n}^{\varepsilon}\right)$ with

$$
\left(u_{n}^{\varepsilon}\right) \subset\left(\Psi_{\varepsilon}\right)_{d_{2}+\tilde{\varepsilon}}^{d}, \quad \Psi_{\varepsilon}^{\prime}\left(u_{n}^{\varepsilon}\right) \underset{n \rightarrow+\infty}{\rightarrow} 0,
$$

where $d=\sup \Phi_{h}\left(V_{2}^{\prime}\right)+1$. For all $k \in \mathbf{N}$ large enough we have $1 / k \in\left(0, \varepsilon_{0}\right]$ and find numbers $n(k) \in \mathbf{N}$ such that $\left(\Psi_{k}:=\Psi_{1 / k}\right)$,

$$
\begin{gathered}
\left\|\Psi_{k}^{\prime}\left(u_{k}\right)\right\| \leqslant 1, \quad u_{k}=u_{n(k)}^{1 / k} \text { and } \\
\left|\left(\Psi_{k}^{\prime}\left(u_{k}\right), u_{k}\right)\right| \leqslant \frac{1}{4 k}\left\|u_{k}\right\| .
\end{gathered}
$$

Observing that $\left(\Psi_{k}\left(u_{k}\right)\right)$ is bounded we find

$$
\begin{aligned}
b\left(u_{k}\right)-( & \left.B u_{k}, u_{k}\right)=a\left(u_{k}\right)+b\left(u_{k}\right)+\left(h, u_{k}\right)-\frac{1}{2 k}\left\|P u_{k}\right\|^{2} \\
& -\left(2 a\left(u_{k}\right)+\left(B u_{k}, u_{k}\right)+\left(h, u_{k}\right)-\frac{1}{k}\left\|P u_{k}\right\|^{2}\right)+a\left(u_{k}\right)-\frac{1}{2 k}\left\|P u_{k}\right\|^{2} \\
= & \Psi_{k}\left(u_{k}\right)-\left(\Psi_{k}^{\prime}\left(u_{k}\right), u_{k}\right)+a\left((\mathrm{Id}-P) u_{k}\right)-\frac{1}{2 k}\left\|P u_{k}\right\|^{2} \\
\leqslant & d+\frac{1}{4 k}\left\|u_{k}\right\|-\frac{1}{2 k}\left\|P u_{k}\right\|^{2}+\text { const } \leqslant \hat{d},
\end{aligned}
$$

for a suitably large constant $\hat{d}<+\infty$ since clearly $\left(\left\|(\operatorname{Id}-P) u_{k}\right\|\right)$ and $\left(a\left((\operatorname{Id}-P) u_{k}\right)\right)$ are uniformly bounded $\left(\left\|\Psi_{k}^{\prime}\left(u_{k}\right)\right\| \leqslant 1\right)$.

Now if $\left\|u_{k}\right\| \rightarrow+\infty$ we infer that $\left\|P u_{k}\right\| \rightarrow+\infty$ while $\left((\operatorname{Id}-P) u_{k}\right)$ is bounded. This implies by our hypotheses a contradiction

$$
+\infty=\lim \left(b\left(u_{k}\right)-\left(B u_{k}, u_{k}\right)\right) \leqslant \hat{d}<+\infty .
$$

Hence $\left(u_{k}\right)$ is bounded. Therefore eventually taking a subsequence

$$
\begin{aligned}
& \Phi_{h}^{\prime}\left(u_{k}\right) \rightarrow 0, \\
& \Phi_{h}\left(u_{k}\right) \rightarrow c \in\left[d_{2}+\tilde{\varepsilon}, d\right], \quad\left(u_{k}\right) \text { bounded. }
\end{aligned}
$$

This implies that $c$ is a critical value. The proof is complete.

The next theorem studies the situation in which $\theta$ is empty. 
THEOREM 6. Assume $\Phi$ verifies ( $\Phi)$. Moreover, suppose that for all sequences $\left(u_{n}\right) \subset H$ with $\left((\mathrm{Id}-P) u_{n}\right)$ bounded and $\left\|P u_{n}\right\| \rightarrow+\infty$ we have

$\lim B u_{n}=0$,

$\lim b\left(u_{n}\right)=\Delta \quad$ where $\Delta \in(0,+\infty)$ is independent of the chosen sequence,

$b(u)<\Delta \quad$ for all $u \in H$.

Then there exists an open neighbourhood $U$ of $X \oplus Z$ such that

(i) for all $h \in \operatorname{cl}(U), \Phi_{h}$ possesses at least one solution;

(ii) for all $h \in U \backslash(X \oplus Z), \Phi_{h}$ possesses at least two solutions;

(iii) the set $\hat{U}=\left\{h \in H \mid \Phi_{h}\right.$ possesses a critical point $\}$ is closed.

Proof. Sketch of the proof. For $h^{*} \in X \oplus Z$ we construct a pair of intersecting sets and show that $\Phi_{h^{*}}$ verifies (WPS) on $\mathbf{R} \backslash\left\{c_{h^{*}}\right\}$, where the constant $c_{h^{*}}$ will be made precise in the proof. ((WPS) on $\mathbf{R} \backslash\left\{c_{h^{*}}\right\}$ means: if $\Phi_{h^{*}}^{\prime}\left(u_{n}\right) \rightarrow 0$ and $\Phi_{h^{*}}\left(u_{n}\right) \rightarrow$ $d \neq c_{h^{*}}$, then $d$ is a critical level.) Theorem 4(i) gives a first solution, also for $\Phi_{h}$ with $h$ in a neighbourhood $V\left(h^{*}\right)$ of $h^{*}$. For $h \in V\left(h^{*}\right) \backslash(X \oplus Z)$ a second pair of intersecting sets will be constructed. Since $\Phi_{h}$ verifies (WPS) the existence of a second critical point follows from Theorem 3 .

Let $A^{-1}: X \oplus Z \rightarrow X \oplus Z$ be the generalized inverse of $A$. For $h \in H$ let $a_{h}(u)=$ $a(u)+(h, u)$. Moreover recall that the compactness of $Q B$ implies that $b \mid x+Z$ is weakly sequentially continuous for all fixed $x$ (see [10, Theorem 8.2, p. 76]).

Step 1. For all $h^{*} \in X \oplus Z$, setting $x_{0}=-A^{-1} P_{-} h, z_{0}=-A^{-1} P_{+} h$, there exists an open neighbourhood $V\left(h^{*}\right)$ such that

$$
\sup \Phi_{h}\left(x_{0}+Z\right)<c_{h}+\Delta
$$

for all $h \in V\left(h^{*}\right)$, where $c_{h}=a_{h}\left(-A^{-1}(\mathrm{Id}-P) h\right)$. Moreover, there exists a constant $\delta>\left\|x_{0}\right\|$ such that

$$
\begin{gathered}
\inf \Phi_{h}\left(z_{0}+W\right)>\sup \Phi_{h}\left(x_{0}+Z\right), \\
W=\{u \in(\operatorname{Id}-Q) H \mid\|u\|=\delta\}
\end{gathered}
$$

for all $h \in V\left(h^{*}\right)$.

Fix $h^{*} \in X \oplus Z$ and let $c_{-}=a_{h^{*}}\left(x_{0}\right)$ and $c_{+}=a_{h^{*}}\left(z_{0}\right)$. Note that at $z_{0} a_{h^{*}} \mid Z$ attains its maximum and that $z_{0}$ is the (unique) solution of $A z+P_{+} h^{*}=0$ in $Z$. Moreover, we have for a suitably large constant $\tau>0$,

$$
\sup \Phi_{h^{*}}\left(x_{0}+Z\right)=\sup \Phi_{h^{*}}\left(x_{0}+\{z \in Z \mid\|z\| \leqslant \tau\}\right) .
$$

We find

$$
\begin{aligned}
\sup \Phi_{h^{*}}\left(x_{0}+Z\right) & =a_{h^{*}}\left(x_{0}\right)+\sup _{Z}\left(a_{h^{*}}(z)+b\left(x_{0}+z\right)\right) \\
& =c_{-}+\sup _{\|z\| \leqslant \tau}\left(a_{h^{*}}(z)+b\left(x_{0}+z\right)\right) \\
& \leqslant c_{-}+\sup a_{h^{*}}(Z)+\sup _{\|z\| \leqslant \tau} b\left(x_{0}+z\right) \\
& \leqslant c_{1}+c_{+}+\bar{b}
\end{aligned}
$$


where $\bar{b}=\sup _{\|z\| \leqslant \tau} b\left(x_{0}+z\right)$. There exists a sequence $\left(z_{n}\right) \subset Z,\left\|z_{n}\right\| \leqslant \tau, z_{n} \rightarrow z$ such that

$$
\bar{b}=\lim b\left(x_{0}+z_{n}\right)=b\left(x_{0}+z\right) .
$$

Hence

$$
\sup \Phi_{h^{*}}\left(x_{0}+Z\right) \leqslant c_{-}+c_{+}+\bar{b}<c_{-}+c_{+}+\Delta .
$$

Next we show that

$$
\sup \Phi_{h^{*}}\left(x_{0}+Z\right)<\inf \Phi_{h^{*}}\left(z_{0}+W\right)
$$

for a suitable constant $\delta>\left\|x_{0}\right\|(W=W(\delta))$. Arguing indirectly we may assume the contrary. Hence there exists a sequence $\left(u_{n}\right) \subset(\operatorname{Id}-Q) H, u_{n}=x_{n}+y_{n}$, with $\left\|u_{n}\right\| \rightarrow+\infty$, such that

$$
\Phi_{h^{*}}\left(z_{0}+u_{n}\right) \leqslant \sup \Phi_{h^{*}}\left(x_{0}+Z\right) \leqslant c_{-}+c_{+}+\bar{b} .
$$

If $\left\|x_{n}\right\| \rightarrow+\infty$, we immediately obtain a contradiction. Therefore we may assume $\left\|y_{n}\right\| \rightarrow+\infty$ and $\left(x_{n}\right)$ bounded. This yields

$$
\begin{aligned}
c_{+}+\inf _{n} a_{h^{*}}\left(x_{n}\right)+\lim b\left(z_{0}+u_{n}\right) & \leqslant \liminf \Phi_{h^{*}}\left(z_{0}+u_{n}\right) \\
& \leqslant c_{-}+c_{+}+\bar{b} .
\end{aligned}
$$

Observing that $c_{-}=\inf a_{h^{*}}(X) \leqslant \inf _{n} a_{h^{*}}\left(x_{n}\right)$ we deduce

$$
\Delta \leqslant \bar{b}<\Delta \text {. }
$$

This contradiction proves (14) for some constant $\delta>\left\|x_{0}\right\|$. Since there exists a constant $c>0$ such that for all $h \in H,\left\|h-h^{*}\right\| \leqslant 1$, we have

$$
\sup \Phi_{h}\left(x_{0}+Z\right)=\sup \Phi_{h}\left(x_{0}+\{z \in Q H \mid\|z\| \leqslant c\}\right)
$$

we find a neighbourhood $V\left(h^{*}\right)$ such that

$$
\begin{gathered}
\sup \Phi_{h}\left(x_{0}+Z\right)<c_{h}+\Delta, \\
\inf \Phi_{h}\left(z_{0}+W\right)>\sup \Phi_{h}\left(x_{0}+Z\right)
\end{gathered}
$$

for all $h \in V\left(h^{*}\right)$ (observe that $c_{h^{*}}=c_{+}+c_{-}$). For all $h^{*} \in X \oplus Z$ we can construct a set having the properties stated in Step 1. Define

$$
U=\bigcup_{h^{*} \in X \oplus Z} V\left(h^{*}\right)
$$

Step 2. Let $h^{*} \in X \oplus Z$ be given and assume $V\left(h^{*}\right), x_{0}, z_{0}, W$, are as constructed in Step 1. Then

(i) for $h \in V\left(h^{*}\right)$ let $\varepsilon_{h}=\frac{1}{4} \min \left\{\inf \Phi_{h}\left(z_{0}+W\right)-\sup \Phi_{h}\left(x_{0}+Z\right), c_{h}+\Delta-\right.$ $\left.\sup \Phi_{h}\left(x_{0}+Z\right)\right\}$. Then there exists for given $h \in V\left(h^{*}\right)$ at least one critical point $u^{\prime}$ with $\Phi_{h}\left(u^{\prime}\right) \leqslant \sup \Phi_{h}\left(x_{0}+Z\right)+\varepsilon_{h}<c_{h}+\Delta$.

(ii) If $h \in V\left(h^{*}\right) \backslash(X \oplus Z)$, there exists a second critical point $u^{\prime \prime}$ with $\Phi_{h}\left(u^{\prime \prime}\right) \geqslant$ $\inf \Phi_{h}\left(x_{0}+W\right)-\varepsilon_{h}>\Phi_{h}\left(u^{\prime}\right)$. 
(i) Let $h \in V\left(h^{*}\right)$ be given. Define $U_{1}=z_{0}+W, U_{2}=z_{0}+\{u \in(\operatorname{Id}-Q) H$ $\|u\| \leqslant \delta\}$. Moreover there exists a constant $\gamma>\left\|z_{0}\right\|$ such that $V_{1}=x_{0}+\{u \in Q H \mid$ $\|u\|=\gamma\}$ and $V_{2}=x_{0}+\{u \in Q H \mid\|u\| \leqslant \gamma\}$ together with $U_{1}$ and $U_{2}$ verify

$$
\begin{aligned}
\inf \Phi_{h}\left(U_{1}\right) & >\sup \Phi_{h}\left(V_{2}\right) \quad(\text { Step 1), } \\
\inf \Phi_{h}\left(U_{2}\right) & >\sup \Phi_{h}\left(V_{1}\right), \\
c_{h}+\Delta & >\sup \Phi_{h}\left(V_{2}\right) \quad(\text { Step 1). }
\end{aligned}
$$

By Proposition 2(ii) (and Lemma 2) we have

$$
\left(U_{2}, U_{1}\right) \stackrel{M}{\sim}\left(V_{2}, V_{1}\right) .
$$

Let $d_{1}=\inf \Phi_{h}\left(U_{2}\right)-\varepsilon_{h}, d_{2}=\sup \Phi_{h}\left(V_{2}\right)+\varepsilon_{h}\left(<c_{h}+\Delta\right)$. Now applying Theorem $4(\mathrm{i})$ we find a sequence $\left(u_{n}\right)$ with

$$
\Phi_{h}^{\prime}\left(u_{n}\right) \rightarrow 0, \quad \Phi_{h}\left(u_{n}\right) \rightarrow d \in\left[d_{1}, d_{2}\right] .
$$

Clearly $\left((\mathrm{Id}-P) u_{n}\right)$ must be bounded. Hence, if $\left\|u_{n}\right\| \rightarrow+\infty$ we must have $\left\|P u_{n}\right\| \rightarrow+\infty$ and find

$$
\begin{aligned}
0 & =\lim \Phi_{h}^{\prime}\left(u_{n}\right)=\lim \left(A u_{n}+B u_{n}+h\right) \\
& =\lim A\left((\operatorname{Id}-P) u_{n}\right)+h .
\end{aligned}
$$

If $P h \neq 0$ we have a contradiction since $R(A)=Y^{\perp}$. Therefore we may assume $P h=0$ or equivalently $h \in Y^{\perp} . A: Y^{\perp} \rightarrow Y^{\perp}$ is a topological isomorphism. Hence, $(\mathrm{Id}-P) u_{n} \rightarrow-A^{-1} h$. We find

$$
\begin{aligned}
d & =\lim \Phi_{h}\left(u_{n}\right)=\lim a_{h}\left((\operatorname{Id}-P) u_{n}\right)+\lim b\left(u_{n}\right) \\
& =a_{h}\left(-A^{-1} h\right)+\Delta \geqslant c_{h}+\Delta>d .
\end{aligned}
$$

Therefore $\left(u_{n}\right)$ must be bounded. By our hypotheses we find a critical point $u^{\prime} \in\left(\Phi_{h}\right)_{d_{1}}^{d_{2}}$ of $\Phi_{h}$.

(ii) Let $h \in V\left(h^{*}\right) \backslash(X \oplus Z)$ and define $U_{1}^{\prime}=\varnothing, U_{2}^{\prime}=U_{1}$, and $e=-P h /\|P h\|$. For $u=x_{0}+z+$ te with $z \in Z, t \in \mathbf{R}^{+}$we have

$$
\Phi_{h}(u)=a_{h}\left(x_{0}\right)+a_{h}(z)+b\left(x_{0}+z+t e\right)-t\|P h\| .
$$

Hence, we find $t_{0}>2 \delta$ such that

$$
\sup \Phi_{h}\left(x_{0}+t_{0} e+Z\right) \leqslant \sup \Phi_{h}\left(x_{0}+Z\right) .
$$

Moreover, from the estimate (15) we infer that for a suitably large number $\beta>\left\|z_{0}\right\|$ we have

$$
\sup \Phi_{h}\left(x_{0}+D\right) \leqslant \sup \Phi_{h}\left(x_{0}+Z\right), \text { where } D=\left\{t e+z \mid t \in\left[0, t_{0}\right],\|z\|=\beta\right\} .
$$

This proves that $\left(U_{2}^{\prime}, U_{1}^{\prime}\right)$ together with $\left(V_{2}^{\prime}, V_{1}^{\prime}\right)$, where

$$
\begin{aligned}
& V_{2}^{\prime}=x_{0}+\left\{t e+z \mid t \in\left[0, t_{0}\right],\|z\| \leqslant \beta\right\}, \\
& V_{1}^{\prime}=\partial V_{2}^{\prime} \quad\left(\text { taken in } x_{0}+\mathbf{R} e \oplus Z\right),
\end{aligned}
$$

verifies

$$
\sup \Phi_{h}\left(V_{1}^{\prime}\right)<\inf \Phi_{h}\left(U_{2}^{\prime}\right), \quad \sup \Phi_{h}\left(V_{2}^{\prime}\right)<\inf \Phi_{h}\left(U_{1}^{\prime}\right)
$$


By Proposition 2(iii) we have

$$
\left(U_{2}^{\prime}, U_{1}^{\prime}\right) \stackrel{M}{\sim}\left(V_{2}^{\prime}, V_{1}^{\prime}\right)
$$

By applying Theorem 4(i) we find a sequence $\left(u_{n}\right)$ with

$$
\begin{aligned}
\Phi_{h}\left(u_{n}\right) \rightarrow \hat{d} & \geqslant \inf \Phi_{h}\left(U_{2}^{\prime}\right)-\varepsilon_{h} \\
& =\inf \Phi_{h}\left(U_{1}\right)-\varepsilon_{h} \\
& >\Phi_{h}\left(u^{\prime}\right) \text { and }
\end{aligned}
$$

Since $P h \neq 0$, the argument used before in (i) shows that $\left(u_{n}\right)$ must be bounded. Hence $\hat{d}$ is a critical value. Since $\hat{d}>\Phi_{h}\left(u^{\prime}\right), \hat{d}$ belongs to a second critical point $u^{\prime \prime}$.

Step 3. $\hat{U}$ is closed.

Let $h \in \operatorname{cl}(\hat{U})$. We have to show that $h \in \hat{U}$. Since $X \oplus Z \subset \operatorname{int}(\hat{U})$ by Step 2, we may assume $h \notin X \oplus Z$. We find a sequence $\left(h_{n}\right) \subset \hat{U}$ with $h_{n} \rightarrow h$ and a corresponding sequence $\left(u_{n}\right) \subset H$ such that $\Phi_{h_{n}}^{\prime}\left(u_{n}\right)=0$. Since $P h \neq 0$ we deduce as in the proof of Step 2 that $\left(u_{n}\right)$ is bounded. Hence, $\left(\Phi_{h_{n}}\left(u_{n}\right)\right)$ is bounded and we may assume, eventually taking a subsequence

$$
\begin{aligned}
& \Phi_{h}\left(u_{n}\right) \rightarrow c, \\
& \Phi_{h}^{\prime}\left(u_{n}\right) \rightarrow 0, \quad\left(u_{n}\right) \text { bounded. }
\end{aligned}
$$

Hence, $c$ is a critical value of $\Phi_{h}$.

Proof of Lemma 3. (i) The convexity of $\theta$ is trivial.

(ii) Let $\bar{h} \in \operatorname{int}(\theta)$. We find $\delta>0$ such that $h \in \operatorname{int}(\theta)$ for all $h \in H$ with $\|h-\bar{h}\| \leqslant \delta$. This implies, for all $y \in Y$,

$$
\liminf _{t \rightarrow+\infty}(b(t y) / t+(h, y)) \geqslant 0 .
$$

For given $y \in Y,\|y\|=1$, taking $h$ such that $(h-\bar{h}, y)=-\delta$, we deduce, making use of the homogeneity of $J_{b}$,

$$
J_{b}(y)+(\bar{h}, y) \geqslant \delta\|y\| .
$$

This shows $J_{b}(y)>-(\bar{h}, y)$ for all $y \in Y \backslash\{0\}$. Conversely, if $J_{b}(y)>-(\bar{h}, y)$ for all $y \in Y \backslash\{0\}$, we deduce by the continuity of $J_{b}$ and the compactness of $\left(\partial B_{1}\right) \cap Y$ the existence of a constant $\delta>0$ with

$$
J_{b}(y)+(\bar{h}, y) \geqslant \delta\|y\| \text { for all } y \in Y .
$$

Now let $h \in H,\|h-\bar{h}\|<\frac{\delta}{2}$. We now show that $h \in \theta$ which completes the proof of (ii). We have $J_{b}(y)+(h, y) \geqslant \frac{\delta}{2}\|y\|$ for all $y \in Y$. Let $\left(y_{n}\right) \subset Y$ with $\left\|y_{n}\right\| \rightarrow$ $+\infty$. Since $\operatorname{dim} Y<+\infty$ we may assume that $\lim y_{n} /\left\|y_{n}\right\|=v$ exists. Let $t_{n}=\left\|y_{n}\right\|$ and $v_{n}=y_{n} /\left\|y_{n}\right\|$. We find since we may assume $t_{n}>0$ for all $n \in \mathbf{N}$

$$
\begin{aligned}
b\left(t_{n} v_{n}\right) / t_{n}-J_{b}\left(y_{n}\right) & =b\left(t_{n} v\right) / t_{n}-J_{b}\left(v_{n}\right)+b\left(t_{n} v_{n}\right) / t_{n}-b\left(t_{n} v\right) / t_{n} \\
& \geqslant b\left(t_{n} v\right) / t_{n}-J_{b}\left(v_{n}\right)-\|B\|\left\|v_{n}-v\right\| .
\end{aligned}
$$


Therefore

$$
\begin{aligned}
\liminf \left(b\left(t_{n} v_{n}\right) / t_{n}-J_{b}\left(v_{n}\right)\right) & \geqslant \liminf \left(b\left(t_{n} v\right) / t_{n}-J_{b}(v)\right) \\
& \geqslant \liminf _{t \rightarrow+\infty}\left(b(t v) / t-J_{b}(v)\right) \\
& =J_{b}(v)-J_{b}(v)=0 .
\end{aligned}
$$

Hence

$$
\begin{aligned}
b\left(y_{n}\right)+\left(h, y_{n}\right) & =J_{b}\left(y_{n}\right)+\left(h, y_{n}\right)+b\left(y_{n}\right)-\left(h, y_{n}\right) \\
& \geqslant \frac{\delta}{2}\left\|y_{n}\right\|+\left(\left(b\left(y_{n}\right)-J_{b}\left(y_{n}\right)\right) /\left\|y_{n}\right\|\right)\left\|y_{n}\right\| \\
& =\left(\frac{\delta}{2}+\varepsilon_{n}\right)\left\|y_{n}\right\| \rightarrow+\infty,
\end{aligned}
$$

where $\liminf \varepsilon_{n}=\liminf \left(\left(b\left(y_{n}\right) /\left\|y_{n}\right\|\right)-J_{b}\left(y_{n} /\left\|y_{n}\right\|\right)\right) \geqslant 0$. This proves (ii).

(iii) If $h \in \operatorname{cl}(\theta)$ there exists for given $\varepsilon>0$ a $\bar{h} \in \theta$ with $\|h-\bar{h}\|<\varepsilon$. We find for $y \neq 0$

$$
\lim _{t \rightarrow+\infty}(b(t y)+(\bar{h}, t y))=+\infty
$$

Therefore

$$
\begin{aligned}
J_{b}(y) & \geqslant-(\bar{h}, y)=(h, y)+(h-\bar{h}, y) \\
& \geqslant-(h, y)-\varepsilon\|y\| .
\end{aligned}
$$

Since $\varepsilon>0$ was arbitrarily chosen

$$
J_{b}(y) \geqslant-(h, y) \text { for all } y \in Y .
$$

(iv) Let $\left(y_{n}\right) \subset Y$ be a sequence with $\left\|y_{n}\right\| \rightarrow+\infty$. If $h \in \tilde{\boldsymbol{\theta}}:=\{h \in H \mid-(h, y)$ $\leqslant J_{b}(y)$ for all $\left.y \in Y\right\}$ we have

$$
J_{b}\left(y_{n}\right)+\left(h, y_{n}\right) \geqslant 0 \text { for all } n \in \mathbf{N} .
$$

This implies

$$
\begin{aligned}
b\left(y_{n}\right)+\left(h, y_{n}\right) & =b\left(y_{n}\right)-J_{b}\left(y_{n}\right)+J_{b}\left(y_{n}\right)+\left(h, y_{n}\right) \\
& \geqslant b\left(y_{n}\right)-J_{b}\left(y_{n}\right) \rightarrow+\infty .
\end{aligned}
$$

Hence, $h \in \theta$. By (iii) $\theta \subset \operatorname{cl}(\theta) \subset \tilde{\theta} \subset \theta$. Therefore $\theta=\operatorname{cl}(\theta)$ and

$$
\theta=\left\{h \in H \mid-(h, y) \leqslant J_{b}(y) \text { for all } y \in Y\right\} .
$$

2. A wave equation problem. Now we apply the abstract results from II, $\S 1$, to a wave equation problem. Consider

$$
\begin{aligned}
& u_{t t}-u_{x x}=c u+g(u)+h \quad \text { in }(0, \pi) \times \mathbf{R}, \\
& u(0, t)=u(\pi, t)=0 \quad \text { for all } t \in \mathbf{R} \text {, }
\end{aligned}
$$
$u$ is $2 \pi$-periodic in $t$.

Here $c>0$ is an eigenvalue of $\square=D_{t}^{2}-D_{x}^{2}$. In (17) we omit a dependence of $g$ on $(x, t)$. However, all the following results remain true for that case under the obvious changes in our hypotheses.

Denote by $C_{\infty}$ the real vector space of arbitrarily often continuously differentiable functions on $(0, \pi) \times \mathbf{R}$, which are $2 \pi$-periodic in $t \in \mathbf{R}$ and verify $u(0, t)=u(\pi, t)$ $=0$ for all $t \in \mathbf{R}$. By $H$ we denote the completion of $C_{\infty}$ with respect to the norm 
$\|u\|=(u, u)^{1 / 2},(u, v)=\int_{C} u v$, where $C=(0, \pi) \times(0,2 \pi)$. Clearly $H \cong L^{2}(C) . H$ possesses a normalized Hilbert basis consisting of eigenfunctions of

$$
\square:\left(\Psi_{n k}\right)_{(n, k) \in \mathbf{N} \times \mathbf{Z}}
$$

with corresponding eigenvalues $n^{2}-k^{2}$. Hence $\square \Psi_{n k}=\left(n^{2}-k^{2}\right) \Psi_{n k}$.

Definition 9. Given $h \in H$, a function $u \in H$ is said to be a weak solution of

$$
\begin{gathered}
\square u=h \quad \text { in }(0, \pi) \times \mathbf{R}, \\
u(0, t)=u(\pi, t)=0 \quad \text { for all } t \in \mathbf{R}, \\
u \text { is } 2 \pi \text {-periodic in } t,
\end{gathered}
$$

iff $(u, \square w)=(h, w)$ for all $w \in C_{\infty}$.

With this definition $\square$ induces a selfadjoint linear operator in $H$ denoted by $\tilde{L}$, having the spectrum $\sigma(\tilde{L})=\left\{n^{2}-k^{2} \mid(n, k) \in \mathbf{N} \times \mathbf{Z}\right\}$ (see $\left.[9,11,12]\right)$ :

$$
\begin{aligned}
\tilde{L} u & =\sum\left(n^{2}-k^{2}\right)\left(u, \Psi_{n k}\right) \Psi_{n k}, \\
D(\tilde{L}) & =\left\{u \in H \mid \sum\left(n^{2}-k^{2}\right)^{2}\left(u, \Psi_{n k}\right)^{2}<+\infty\right\} .
\end{aligned}
$$

The following is well known.

LEMMA 4. The following statements are equivalent:

(i) $u$ is a weak solution of (18).

(ii) $\tilde{L} u=h$.

Introduce an operator $L$ by

$$
\begin{gathered}
L=\tilde{L}-c \mathrm{Id}, \quad D(L)=D(\tilde{L}), \\
c>0 \text { an eigenvalue of } \tilde{L} .
\end{gathered}
$$

We have

(L) $L: H \supset D(L) \rightarrow H$ is a selfadjoint operator with a nontrivial kernel $N(L)$, $\operatorname{dim} N(L)<+\infty$, such that the functions in $N(L)$ have the unique continuation property, i.e. if $u \in N(L)$ and meas $(\{d \in C \mid u(d)=0\})>0$ then $u \equiv 0$. Moreover, $\sigma(L) \backslash\{-c\}$ contains only in $\mathbf{R}$ isolated eigenvalues having a finite multiplicity.

Assume

(F0) $g: \mathbf{R} \rightarrow \mathbf{R}$ is continuous and has bounded range. Moreover, $s \rightarrow g(s)+c s$ is nondecreasing on $\mathbf{R}$.

Then $g$ induces a continuous potential operator $F: H \rightarrow H:(F u)(x, t)=g(u(x, t))$ having a bounded range and a normalized potential $f \in C^{1}(H, \mathbf{R}), f(0)=0$, defined by

$$
f(u)=\int_{C} G(u), \quad G(s)=\int_{0}^{s} g(m) d m .
$$

Observe that the weak solutions of (17), i.e. the functions $u \in H$ verifying

$$
(u, \square w)=(c u+g(u)+h, w) \text { for all } w \in C_{\infty},
$$

are exactly the solutions of the operator equation

$$
L u=F u+h .
$$


Before we give a statement of the results we shall show that (19) is equivalent to a critical point problem for a functional $\Phi \in C^{1}(H, \mathbf{R})$ verifying $(\Phi)$.

Following Amann [3] denote by $\left(E_{\lambda}\right)_{\lambda \in \mathbf{R}}$ the spectral resolution of $L$ and define orthogonal projections by

$$
P_{-}=\int_{-\infty}^{0-} d E_{\lambda}, \quad P=\int_{0-}^{0+} d E_{\lambda}, \quad P_{+}=\int_{0+}^{+\infty} d E_{\lambda}
$$

and corresponding mutually orthogonal subspaces $X, Y, Z$ by

$$
X=P_{-} H, \quad Y=P H, \quad Z=P_{+} H .
$$

Clearly $H=X \oplus Y \oplus Z$. Define bounded linear selfadjoint operators by

$$
R=\int_{-\infty}^{0-}(-\lambda)^{-1 / 2} d E_{\lambda}, \quad S=\int_{0+}^{+\infty} \lambda^{-1 / 2} d E_{\lambda} .
$$

Since 0 is an isolated point of the spectrum, both operators are well defined and bounded. Moreover, $S$ is compact because $\sigma(L) \cap \mathbf{R}^{+}$contains only in $\mathbf{R}$ isolated points having finite multiplicity. Define $\Phi \in C^{1}(H, \mathbf{R})$ by

$$
\Phi(u):=a(u)+b(u):=\frac{1}{2}\left(\left(P_{-}-P_{+}\right) u, u\right)+f((R+P+S) u)
$$

and let $\bar{\Phi}_{h}=\Phi+((R+P+S) h, \cdot)=\Phi_{(R+P+S) h}$. If $u$ is a critical point of $\bar{\Phi}_{h}$ we find

$$
\left(P_{-}-P_{+}\right) u+(R+P+S)(F(R+P+S) u+h)=0 .
$$

Applying $-R+P+S$ we obtain for $w=(R+P+S) u$

$$
-(\mathrm{Id}-P) w+\left(S^{2}-R^{2}+P\right)(F w+h)=0 .
$$

Observing that $S^{2}-R^{2}+P=(L+P)^{-1}$ we find

$$
L w=F w+h .
$$

Hence, in order to find weak solutions of the wave equation problem it is enough to study $\bar{\Phi}_{h}$. Observe that $P_{+} B=S F(R+P+S)$ is compact since $S$ is compact. If we can show that, for all $h \in H, \Phi_{h} \in \Sigma_{*}$ we know by the preceding discussion that $(\Phi)$ is verified.

Lemma 5. Let $\Phi$ be as defined in (20) and suppose that (F0) holds. Then, if, for a bounded sequence $\left(u_{n}\right) \subset H$, we have

$$
\Phi_{h}^{\prime}\left(u_{n}\right) \rightarrow 0 \text { and } \Phi_{h}\left(u_{n}\right) \rightarrow d,
$$

$d$ is a critical value. Hence $\Phi_{h} \in \Sigma_{*}=\Sigma_{*}(H, A, Q)$.

Proof. Let $\chi_{n}=\Phi_{h}^{\prime}\left(u_{n}\right)$. We have

$$
-\chi_{n}+\left(P_{-}-P_{+}\right) u_{n}+(R+P+S)\left(F(R+P+S) u_{n}\right)+h=0 \text {. }
$$

Denote by $N: H \rightarrow N(\tilde{L})$ the orthogonal projection. It is easy to show that $(\mathrm{Id}-N)(R+P+S)$ is compact since $\tilde{L}^{-1}: N(\tilde{L})^{\perp} \rightarrow N(\tilde{L})^{\perp}$ is compact. This implies that $\left((\operatorname{Id}-N) u_{n}\right)$ is relatively compact because $\chi_{n} \rightarrow 0$ and $\operatorname{dim} N(L)<$ $+\infty$. By the reflexivity of $H$ and the preceding discussion we may assume

$$
(\operatorname{Id}-N) u_{n} \rightarrow u^{1} \in N(\tilde{L})^{\perp}, \quad N u_{n} \rightarrow u^{2} \in N(\tilde{L}) .
$$


Let $w_{n}=(R+P+S) u_{n}$ and $w=(R+P+S)\left(u^{1}+u^{2}\right)$. We infer

$$
(\mathrm{Id}-N) w_{n} \rightarrow(\mathrm{Id}-N) w \text { and } N w_{n} \rightarrow N w \text {. }
$$

Let $h^{*}=(-R+P+S) h$. Applying $-R+P+S$ to (21), observing that $-R^{2}+S^{2}$ $+P=(L+P)^{-1}$ we infer

$$
-(-R+P+S) \chi_{n}-(\mathrm{Id}-P) w_{n}+(L+P)^{-1} F w_{n}+h^{*}=0 .
$$

This implies

$$
(L+P)\left((\mathrm{Id}-P) w_{n}+(-R+P+S) \chi_{n}-h^{*}\right)=F w_{n} .
$$

Hence

$$
L\left(w_{n}+(-R+P+S) \chi_{n}-h^{*}\right)=F w_{n}+P h^{*}-P \chi_{n} .
$$

Since the right-hand side is bounded we deduce

$$
L\left(w_{n}+(-R+P+S) \chi_{n}-h^{*}\right)-L\left(w-h^{*}\right) .
$$

And obviously

$$
\tilde{L}\left(w_{n}+(-R+P+S) \chi_{n}-h^{*}\right)-\tilde{L}\left(w-h^{*}\right) .
$$

Let $q \in H$ be arbitrarily chosen. By the monotonicity of $c$ Id $+F$ we find

$$
\begin{aligned}
&\left(\tilde{L}\left(w_{n}+(-R+P+S) \chi_{n}-h^{*}\right), w_{n}-q\right) \\
& \geqslant\left(c q+F q+P h^{*}-c h^{*}, w_{n}-q\right)-\mathrm{const}\left\|\chi_{n}\right\|,
\end{aligned}
$$

where the constant is only depending on $q$. We can take the limit, observing that

$$
\begin{aligned}
\left(\tilde { L } \left(w_{n}+(-R+P+\right.\right. & \left.\left.S) \chi_{n}-h^{*}\right), w_{n}-q\right) \\
& =\left(\tilde{L}\left(w_{n}+(-R+P+S) \chi_{n}-h^{*}\right),(\operatorname{Id}-N)\left(w_{n}-q\right)\right) .
\end{aligned}
$$

Hence

$$
\left(\tilde{L}\left(w-h^{*}\right), w-q\right) \geqslant\left(c q+F q+P h^{*}-c h^{*}, w-q\right) .
$$

Now choosing $q=w-t q^{\prime}$, for $t>0$ and $q^{\prime} \in H$ arbitrary, we find, dividing by $t$ and taking the limit $t \rightarrow 0$,

$$
\left(\tilde{L}\left(w-h^{*}\right)-c w-F w-P h^{*}+c h^{*}, q^{\prime}\right) \geqslant 0 .
$$

Therefore

$$
L\left(w-h^{*}\right)-F w-P h^{*}=0 .
$$

This yields

$$
w-h^{*}-(L+P)^{-1} F w-P w=0 .
$$

Observing that $w=(R+P+S)\left(u^{1}+u^{2}\right)$ and $h^{*}=(-R+P+S) h$ we obtain, by the injectivity of the operator $-R+P+S$ for $u_{0}=u^{1}+u^{2}$,

$$
\left(P_{-}-P_{+}\right) u_{0}+B u_{0}+h=0 \text {. }
$$

Hence

$$
\Phi_{h}^{\prime}\left(u_{0}\right)=0
$$


It remains to show that $\Phi_{h}\left(u_{0}\right)=d$. Since $c$ Id $+F$ is monotone and a gradient we have

$$
\begin{aligned}
\frac{c}{2}\|w\|^{2}+f(w)-\frac{c}{2}\left\|w_{n}\right\|^{2}-f\left(w_{n}\right) \geqslant\left(c w_{n}+F w_{n}, w-w_{n}\right) & \\
=\left(\tilde { L } \left(w_{n}+\right.\right. & \left.(-R+P+S) \chi_{n}-h^{*}\right) \\
& \left.-c\left((-R+P+S) \chi_{n}-h^{*}\right)-P h^{*}+P \chi_{n}, w-w_{n}\right) .
\end{aligned}
$$

As before it follows that the right-hand side converges to 0 . Hence

$$
\frac{c}{2}\|w\|^{2}+f(w) \geqslant \lim \sup \left(\frac{c}{2}\left\|w_{n}\right\|^{2}+f(w)\right) .
$$

On the other hand, by the convexity,

$$
\frac{c}{2}\|w\|^{2}+f(w) \leqslant \liminf \left(\frac{c}{2}\left\|w_{n}\right\|^{2}+f\left(w_{n}\right)\right) .
$$

Therefore

$$
\lim \left(\frac{c}{2}\left\|w_{n}\right\|^{2}+f\left(w_{n}\right)\right)=\frac{c}{2}\|w\|^{2}+f(w) .
$$

Now since for $\Psi(u)=\frac{1}{2}\left\|P_{-} u\right\|^{2}-\frac{1}{2}\left\|P_{+} u\right\|^{2}-\frac{c}{2}\|(R+P+S) u\|^{2}+(h, u)$ we have

$$
\Psi(u)=\Psi((\mathrm{Id}-N) u)+(h, N u)
$$

we find, observing that $(\operatorname{Id}-N) u_{n} \rightarrow(\operatorname{Id}-N) u_{0}$,

$$
\begin{aligned}
\lim \Psi\left(u_{n}\right) & =\lim \Psi\left((\operatorname{Id}-N) u_{n}\right)+\lim \left(h, N u_{n}\right) \\
& =\Psi\left((\operatorname{Id}-N) u_{0}\right)+\left(h, N u_{0}\right)=\Psi\left(u_{0}\right) .
\end{aligned}
$$

Therefore

$$
\begin{aligned}
\lim \Phi_{h}\left(u_{n}\right) & =\lim \left(\Psi\left(u_{n}\right)+f\left((R+P+S) u_{n}\right)+\frac{c}{2}\left\|(R+P+S) u_{n}\right\|^{2}\right) \\
& =\lim \Psi\left(u_{n}\right)+\lim \left(f\left(w_{n}\right)+\frac{c}{2}\left\|w_{n}\right\|^{2}\right) \\
& =\Psi\left(u_{0}\right)+f(w)+\frac{c}{2}\|w\|^{2} \\
& =\Psi\left(u_{0}\right)+f\left((R+P+S) u_{0}\right)+\frac{c}{2}\left\|(R+P+S) u_{0}\right\|^{2} \\
& =\Phi_{h}\left(u_{0}\right),
\end{aligned}
$$

which implies $\Phi_{h}\left(u_{0}\right)=d$.

In order to apply Theorem 5 we impose the following hypotheses on $g$ :

(F1) (i) $\gamma_{ \pm}=\lim _{s \rightarrow \pm \infty} G(s) /|s|$ exist in $\mathbf{R}^{+}$,

(ii) $\lim _{s \rightarrow \pm \infty}\left(G(s)-\gamma_{ \pm}|s|\right)=+\infty$,

(iii) $\lim _{|s| \rightarrow+\infty}(G(s)-g(s) s)=+\infty$.

Observe that (i) and (ii) imply that $G(s) \rightarrow+\infty$ for $|s| \rightarrow+\infty$.

Lemma 6. Assume $g$ verifies (F0) and (F1) and let $P, B$, and $b$ be as defined before.

Then we have for all sequences $\left(u_{n}\right) \subset H$ with $\left((\mathrm{Id}-P) u_{n}\right)$ bounded, $\left\|P u_{n}\right\| \rightarrow+\infty$,

$$
\begin{aligned}
\lim \left(b\left(u_{n}\right)-J_{b}\left(P u_{n}\right)\right) & =+\infty, \\
\lim \left(b\left(u_{n}\right)-\left(B u_{n}, u_{n}\right)\right) & =+\infty
\end{aligned}
$$

and

$$
J_{b}(y)=\lim _{t \rightarrow+\infty} b(t y) / t=\gamma_{+} \int_{C} y_{+}+\gamma_{-} \int_{C} y_{-}, \quad y \in Y,
$$

where $y_{+}=\max (0, y)$ and $y_{-}=-\min (0, y)$. 
Hence, if $g$ verifies (F0) and (F1) we know by the preceding discussion that $\Phi$ defined in (20) fulfills the hypotheses of Theorem 5. As an immediate consequence we have the following result:

TheOrem 7. Assume $g$ verifies (F0) and (F1). Define the set $\theta$ by $\theta=\{h \in H \mid$ $\left.-(h, y) \leqslant J_{b}(y) \forall y \in Y\right\}$. Then there exists an open set $U$ containing $\theta$ such that

(i) for all $h \in U$ (17) possesses at least one weak solution;

(ii) for all $h \in U \backslash \theta(17)$ possesses at least two weak solutions.

Note that all results remain true under the obvious changes if $g$ is depending on $x$ and $t$.

Theorem 7 extends existence and multiplicity results given in [12] for the wave equation problem. There it was assumed in contrast to (F1) that $g_{ \pm}=\lim _{s \rightarrow \pm \infty} g(s)$ exist in $\mathbf{R}$ and $g_{-} \leqslant 0 \leqslant g_{+}, g(s) \geqslant g_{+}, g(s) \leqslant g_{-}$for all $s \geqslant s_{0}$ and $s \leqslant-s_{0}$, respectively, for a suitable constant $s_{0}>0$, and $\liminf _{s \rightarrow \pm \infty}\left(g(s)-g_{ \pm}\right) s>0$. Observe that here it is not necessary that $g_{+}$and $g_{-}$exist. Moreover, there are examples in which the second hypothesis is not verified but (F1) holds. Further, the third hypothesis implies that $g(s)$ tends to $g_{+}$and $g_{-}$not more rapidly than $|s|^{-1}$. For example $g(s)=2 s\left(1+s^{2}\right)^{-1} \ln \left(1+s^{2}\right)^{-1}$ verifies (F1) but not the hypothesis of [12] $\left(G(s)=\ln \left(\ln \left(1+s^{2}\right)\right)\right)$. Note, however, that the abstract results proved in [12] apply also to the nonvariational case and are obtained by degree arguments in connection with an approximation procedure, in contrast to the variational arguments used here.

Proof of Theorem 7. By the preceding discussion, $\Phi$ verifies the hypotheses of Theorem 5. Hence there exists an open set $U^{*}$ containing $\theta$ such that (i) and (ii) of Theorem 5 hold. The mapping $T: H \rightarrow H: T h=(R+P+S) h$ is injective and continuous. Therefore $T^{-1}\left(U^{*}\right)=U$ is open and moreover we have $T^{-1}(\theta)=\theta$ using that $\theta=X \oplus P \theta \oplus Z$ and $T P=P T=P$.

Let $h \in U$. Then $T h \in U^{*}$ and we find a critical point $u_{1}$ of $\Phi_{T h}$ which is a weak solution of (17). If $h \in U \backslash \theta$ we have $T h \in U^{*} \backslash \theta$ and find a second weak solution.

It remains to prove Lemma 6 which we have used implicitly in the proof of Theorem 7.

Proof of Lemma 6. Let $y \in Y=N(L), y \neq 0$. We have, for $t>0, b(t y) / t=$ $\int_{C} G(t y) / t$ and $|G(t y) / t| \leqslant$ const. By Lebesgue's Theorem using our hypotheses

$$
\begin{aligned}
J_{b}(y) & =\liminf _{t \rightarrow+\infty} b(t y) / t=\lim _{t \rightarrow+\infty} b(t y) / t \\
& =\int \lim _{t \rightarrow+\infty} G(t y) / t=\int\left(\gamma_{+} y_{+}+\gamma_{-} y_{-}\right) .
\end{aligned}
$$

If $\left(u_{n}\right)$ is as in our hypotheses, the sequence $\left(w_{n}\right)=\left((R+P+S) u_{n}\right)$ has the same properties since $P u_{n}=P w_{n}$. Passing to a subsequence, if necessary, we may assume

$$
\begin{gathered}
w_{n} /\left\|w_{n}\right\| \rightarrow w, \quad P w_{n} /\left\|P w_{n}\right\| \rightarrow w, \\
\left(w_{n} /\left\|w_{n}\right\|\right)(x, t) \rightarrow w(x, t) \quad \text { for a.a. }(x, t) \in C, \\
\left(P w_{n} /\left\|P w_{n}\right\|\right)(x, t) \rightarrow w(x, t) \quad \text { for a.a. }(x, t) \in C .
\end{gathered}
$$


Since $\|w\|=1$ and $w \in N(L)$ we infer by the unique continuation property $\operatorname{meas}([w \neq 0] \cap C)=\operatorname{meas}(C)$ and

$$
\begin{gathered}
P w_{n}(x, t) \rightarrow \begin{cases}+\infty & \text { on }[w>0], \\
-\infty & \text { on }[w<0]\end{cases} \\
w_{n}(x, t) \rightarrow \begin{cases}+\infty & \text { on }[w>0], \\
-\infty & \text { on }[w<0]\end{cases}
\end{gathered}
$$

Moreover, for $v_{n}=P w_{n}$,

$$
\begin{aligned}
b\left(u_{n}\right)-J_{b}\left(P u_{n}\right) & =f\left(w_{n}\right)-J_{b}\left(P w_{n}\right) \\
& =f\left(v_{n}\right)-J_{b}\left(v_{n}\right)+f\left(w_{n}\right)-f\left(v_{n}\right) \\
& \geqslant f\left(v_{n}\right)-J_{b}\left(v_{n}\right)-\left\|F \sup _{n}\right\|(\operatorname{Id}-P) u_{n} \| \\
& =f\left(v_{n}\right)-J_{b}\left(v_{n}\right)+\text { const. }
\end{aligned}
$$

We have, therefore

$$
\begin{aligned}
b\left(u_{n}\right)-J_{b}\left(P u_{n}\right) \geqslant & \int_{C}\left(G\left(v_{n}\right)-\gamma_{+} v_{n}\right) \chi_{\left[v_{n}>0\right]} \\
& +\left(G\left(v_{n}\right)-\gamma_{-} v_{n}\right) \chi_{\left[v_{n}<0\right]}-\text { const } \\
\equiv & \int_{C}\left(a_{n}^{+}+a_{n}^{-}\right)-\text {const. }
\end{aligned}
$$

By our hypotheses, $a_{n}^{+} \rightarrow+\infty$ on $[w>0]$ and $a_{n}^{-} \rightarrow+\infty$ on $[w<0]$. By Fatou's Lemma

$$
\lim \left(b\left(u_{n}\right)-J_{b}\left(P u_{n}\right)\right)=+\infty
$$

Now observe that

$$
b\left(u_{n}\right)-\left(B u_{n}, u_{n}\right)=f\left(w_{n}\right)-\left(F w_{n}, w_{n}\right) .
$$

Again by Fatou's Lemma $\lim \left(b\left(u_{n}\right)-\left(B u_{n}, u_{n}\right)\right)=+\infty$.

Next we study the case where $G$ has bounded range. The result will be an easy application of Theorem 6 . We impose the following hypotheses on $g$ :

(F2) (i) $\lim _{s \rightarrow \pm \infty} g(s)=0$;

(ii) $\lim _{s \rightarrow+\infty} G(s)=\lim _{s \rightarrow-\infty} G(s)=G_{\infty} \in \mathbf{R}^{+}$;

(iii) $G_{\infty}>G(s)$ for all $s \in \mathbf{R}$.

We need, as before, a lemma which shows that $b$ and $B$ verify the hypotheses of Theorem 6 . The proof of this lemma is similar but easier than the proof of Lemma 6 and is left to the reader.

Lemma 7. Assume $g$ verifies (F0) and (F2) and let $B$ and $b$ be as defined before. Then we have for all sequences $\left(u_{n}\right) \subset H$ with $\left((\mathrm{Id}-P) u_{n}\right)$ bounded, $\left\|P u_{n}\right\| \rightarrow+\infty$,

$$
\lim B u_{n}=0 \text {, }
$$

$$
\begin{gathered}
\lim b\left(u_{n}\right)=\Delta=\int_{C} G_{\infty}=G_{\infty} \operatorname{meas}(C), \\
b(u)<\Delta \quad \text { for all } u \in H .
\end{gathered}
$$


We have the following theorem:

THEOREM 8. Assume $g$ verifies (F0) and (F2). Then there exists an open neighbourhood $U$ of $N(L)^{\perp}$ such that

(i) (17) possesses for $h \in \mathrm{cl}(U)$ at least one weak solution,

(ii) (17) possesses for $h \in U \backslash N(L)^{\perp}$ at least two weak solutions,

(iii) $R(L-F)$ is closed.

Proof. By the preceding discussion $\Phi$ verifies the hypotheses of Theorem 6. As in the proof of Theorem 7 we immediately find that (i) and (ii) hold ((i) is proved for the case $h \in U)$. It remains to show (iii). By Theorem 6(iii), $\hat{U}=\left\{h \in H \mid \Phi_{h}\right.$ possesses a critical point $\}$ is closed. Now observe that $T^{-1}(\hat{U})=R(L-F)$. Since $T$ is continuous and $\hat{U}$ closed (iii) follows.

Results similar to Theorems 7 and 8 hold for (variational) elliptic problems without the monotonicity assumption on $g+c \mathbf{I d}_{\mathbf{R}}$. In that case we obtain strong extensions of results given in $[13,14]$. For example we can treat the problem $-\Delta u=\lambda_{n} u+g(u)+h$ in $\Omega, u=0$ on $\partial \Omega$, where $g(s)=s\left(1+|s|^{\sigma}\right)^{-1}, \sigma>1$, which could not be treated in [13] for $\sigma>2$ and could be studied in [14] only for $\sigma \leqslant 3$ and $\lambda_{n}=\lambda_{1}$. Clearly Theorem 8 is also an extension of results given in [12].

\section{REFERENCES}

1. P. Rabinowitz, Some minimax theorems and applications to nonlinear partial differential equations, Nonlinear Analysis (L. Cesari, R. Kannan and H. F. Weinberger, eds.), Academic Press, New York.

2. __ Free vibrations for a semilinear wave equation, Comm. Pure Appl. Math. 31 (1978), 31-68.

3. H. Amann, Saddle points and multiple solutions of differential equations, Math. Z. 169 (1979), $127-166$.

4. H. Brézis, J. M. Coron and L. Nirenberg, Free vibrations for a nonlinear wave equation and a theorem of P. Rabinowitz, Comm. Pure Appl. Math. 33 (1980), 667-684.

5. I. Ekeland and J. M. Lasry, On the number of periodic trajectories for a Hamiltonian flow on a convex energy surface, Ann. of Math. (2) 112 (1980), 283-319.

6. V. Benci and P. Rabinowitz, Critical point theory for indefinite functionals, Invent. Math. 52 (1979), $241-273$

7. R. Palais, Critical point theory and the minimax-principle, Proc. Sympos. Pure Math., vol. 15, Amer. Math. Soc., Providence, R. I., 1970, pp. 185-212.

8. P. Hess, On a theorem of Landesman and Lazer, Indiana Univ. Math. J. 23 (1974), 827-829.

9. H. Brézis and L. Nirenberg, Characterizations of the ranges of some nonlinear operators and applications to boundary value problems, Ann. Scuola Norm. Sup. Pisa 4 (1978), 225-326.

10. M. M. Vainberg, Variational methods for the study of nonlinear operators, Holden-Day, San Francisco, Calif, 1964.

11. H. Hofer, On the range of a wave operator with a nonmonotone nonlinearity, Math. Nachr. (to appear).

12. Multiplicity result for a class of nonlinear problems, with application to a wave equation problem, J. Nonlinear Analysis 5 (1981), 1-11.

13. P. Hess, Nonlinear perturbations of linear elliptic and parabolic boundary value problems at resonance: existence of multiple solutions, Ann. Scuola Norm. Sup. Pisa 5 (1978), 527-537.

14. S. Fucik and P. Hess, Nonlinear perturbations of linear operators having nullspace with strong unique continuation property, J. Nonlinear Analysis 3 (1979), 271-277.

15. A. Ambrosetti and P. Rabinowitz, Dual variational methods in critical point theory with applications, J. Funct. Anal. 14 (1973), 349-381.

MAThematisches INSTitut DER UNIVERSität ZÚrich, RÄMistrasse 74, 8006 ZURich, SwitzerLAND 